IASSNS-HEP-94/90

\title{
Fractional Statistics in One-Dimension: View From An Exactly Solvable Model
}

\author{
Z. N. C. Ha \\ School of Natural Sciences, Institute for Advanced Study, \\ Princeton, New Jersey 08540
}

(October 25, 1994)

\begin{abstract}
One-dimensional fractional statistics is studied using the Calogero-Sutherland model (CSM) which describes a system of non-relativistic quantum particles interacting with inverse-square two-body potential on a ring. The inversesquare exchange can be regarded as a pure statistical interaction and this system can be mapped to an ideal gas obeying the fractional exclusion and exchange statistics. The details of the exact calculations of the dynamical correlation functions for this ideal system is presented in this paper. An effective low-energy one-dimensional "anyon" model is constructed; and its correlation functions are found to be in agreement with those in the CSM; and this agreement provides an evidence for the equivalence of the first- and the second-quantized construction of the 1D anyon model at least in the long wave-length limit. Furthermore, the finite-size scaling applicable to the conformally invariant systems is used to obtain the complete set of correlation exponents for the CSM.
\end{abstract}

PACS 05.30-d, 71.10.+x, 71.27.+a, 73.40.H 


\section{INTRODUCTION}

The fractional statistics in low-dimensional $(<3)$ quantum systems had been a subject appreciated only by a few [四,2]; however, the discovery of the quantum Hall effect [3] has perhaps changed the status of the subject forever, promoting and reshaping it into one of the most profound as well as popular field in modern physics and into a necessary conceptual tool in condensed matter physics.

The fractional statistics is usually discussed in the context of two-dimensional systems where the adiabatic transport of a test particle around the others can be used to determine the statistics independent of the dynamical nature of the interacting system. In onedimension (1D), however, the dynamics and the kinematics can not be decoupled in an unambiguous way (i.e., an exchange necessarily involves a scattering), and the assignment of the statistics to 1D particles is largely a matter of taste. On the other hand, a good taste will yield fruitful concepts and tools.

In this paper I study the 1D fractional statistics using a specific model called CalogeroSutherland Model (CSM) [4] which describes a systems of non-relativistic quantum particles interacting with a pairwise potential that falls off as inverse-square of the distance between the particles. One of the most important practical features of this model is that the inversesquare potential can be regarded as a pure statistical interaction and the model maps to an ideal gas of particles obeying the fractional statistics [5],6]. Usually, the first step in understanding a general class of interacting Fermi system known as Fermi liquid is to study the ideal Fermi gas which gives rise to important concepts and tools such as the Fermi surface. Much in the same spirit I start with the simplest 1D system (i.e., the CSM) obeying the fraction statistics.

First, I list here some of the recent developments. A lattice cousin of the CSM called Haldane-Shastry Model (HSM), which corresponds to the $S U(2)$ Heisenberg spin chain with the inverse-square instead of the usual nearest neighbor exchange, has triggered a surge of interest in this class of models [7]. The HSM is known to possess the quantum symmetry 
algebra known as Yangian [8] and can be considered as a model of ideal $S U(2)$ spinon gas obeying the semionic fractional statistics [9]. The $S U(n)$ versions of the CSM [10] and the HSM [10,11] now exist and in particular the spectrum of $S U(n)$ HSM is known to possess the Bethe ansatz string structure [12]. The list goes on, but I concentrate on the $U(1)$ CSM in this paper.

The CSM is intimately related to the circular ensembles in random matrix theory first introduced by Freeman Dyson [13]. In particular, the eigenvalue distribution functions for the orthogonal, unitary, and symplectic random matrices correspond to the ground state wavefunctions of the CSM at the interaction parameters $\lambda=1 / 2,1$ and 2, respectively. ( $\lambda=1$ case corresponds to the free Fermi gas.) Some static correlation functions of the CSM can be calculated using the techniques developed for the random matrices [14].

More recently, Simons et. al. have been successful in mapping the CSM to the matrix model and to the non-linear sigma model where the supersymmetric algebra is applicable [15], and therefore are able to calculate the dynamical density-density correlation functions (DDDCF) for the CSM at $\lambda=1 / 2,1$ and 2 [16]. Haldane and Zirnbauer using the similar method calculate the one-particle Green's function at $\lambda=2$ (i.e., the symplectic case) [17]. Forrester [18] also calculates some static correlation functions at integer interaction parameters using a generalized form of the celebrated Selberg integral formula 19.

It turns out that the eigenstates of the CSM can be written in terms of Jack polynomials [5.20] whose known algebraic properties provide a powerful and direct method for calculating the most general correlation functions. Recently, the author has been successful in calculating the exact DDDCF and one-particle Green's function at arbitrary rational interaction parameters [6]. The method employed is new and is one of the main subjects in this paper.

The exact calculations of the correlation functions further provide conclusive evidences of the inherent fractional exclusion and exchange statistics embodied in the CSM [6]. I also make a direct connection of this model to the edge states of the fractional quantum Hall droplet by first constructing an effective low-energy one-dimensional "anyon" fluid model based on a general gauge invariance argument, and then showing that the dynamical 
correlation functions agree with those of the CSM. This connection between the CSM and the edge states has previously been suggested by various people [21].

This paper is organized in the following way. In Section II I show how to construct general eigenstates of the CSM, and in Section [II] some properties of the Jack polynomials are introduced. In Section $\square$ some key aspects of the exclusion and the exchange statistics are discussed. I show how to calculate the DDDCF and the one-particle Green's function in Section $\mathrm{V}$ and V1. In Section VII the "harmonic-fluid" description of the anyon fluid is constructed and the dynamical correlation functions calculated; and I further show that they agree with those of the CSM. This agreement provides an explicit connection between the CSM and the system of coupled left- and right-edges of the fractional quantum Hall effect and further shows the equivalence between the first- and the second-quantized construction of one-dimensional "anyon" gas at least in the long-wavelength limit. In Section VIII the finite-size scaling as applied to the conformally invariant systems is used to obtain the complete set of correlation exponents. I also discuss some aspect of the lattice cousins of the CSM in Section IX].

\section{EIGENSTATES OF CALOGERO-SUTHERLAND MODEL}

In this section I introduce the CSM and show how to construct the general eigenstates of the model following Sutherland [22]. First, the Hamiltonian for the CSM on a ring of length $L$ is given by

$$
H=-\sum_{j=1}^{N} \frac{\partial^{2}}{\partial x_{j}^{2}}+\sum_{j<l} \frac{2 \lambda(\lambda-1)}{d^{2}\left(x_{j}-x_{l}\right)}
$$

where $\hbar^{2} / 2 m=1$ and $d(x)=(L / 2 \pi)|\sin (\pi x / L)|$. The ground state wavefunctions of the model at integer values of $\lambda$ corresponds to 1D versions of Laughlin's wavefunctions [23] and are given by

$$
\Psi_{0}=\prod_{i<j}\left(z_{i}-z_{j}\right)^{\lambda} \prod_{k} z_{k}^{J_{0}}
$$


where the current $J_{0}=-\lambda(N-1) / 2$ and $z_{j}=\exp \left(i 2 \pi x_{j} / L\right)$. When $0<\lambda<1$ there is another possible ground state with power $1-\lambda$; however, only the solution with power $\lambda$ will be considered for reasons of continuity with $\lambda>1$ solutions.

The excited states of this model are constructed by multiplying some symmetric polynomials to the ground state wavefunction, and this construction is analogous to that of the gapless edge excitations of the quantum Hall effect [24. A general excited state $\Psi_{\mathbf{n}}^{\lambda}=\Psi_{0} J_{\mathbf{n}}^{\lambda}$ is labeled by the quantum numbers $\mathbf{n}=\left(n_{1}, n_{2}, \ldots, n_{N}\right)$, and $J_{\mathbf{n}}^{\lambda}$ satisfies the following new eigenvalue equation

$$
\tilde{H} J_{\mathbf{n}}^{\lambda}=E_{\mathbf{n}} J_{\mathbf{n}}^{\lambda}
$$

where $\tilde{H}=H_{0}+\lambda H_{1}$, and

$$
\begin{aligned}
& H_{0}=\sum_{j=1}^{N}\left(z_{j} \partial_{z_{j}}\right)^{2}, \\
& H_{1}=\sum_{j<k} \frac{z_{j}+z_{k}}{z_{j}-z_{k}}\left(z_{j} \partial_{z_{j}}-z_{k} \partial_{z_{k}}\right) .
\end{aligned}
$$

The eigenstates of the new Hamiltonian $\tilde{H}$ are represented in terms of the following bosonic basis states

$$
\Phi(\mathbf{n})=\sum_{P} \prod_{j=1}^{N} z_{j}^{n_{P_{j}}},
$$

where the sum extends over all permutations of the integer set $\mathbf{n}$ which can be considered as a set of bosonic quantum numbers with no restrictions on their values. Since $\Phi(\mathbf{n})$ does not depend on the ordering of the quantum numbers, let $n_{1} \geq n_{2} \geq \ldots \geq n_{N}$ without loss of generality. These symmetric polynomials form a complete basis.

The action of $\tilde{H}$ on $\Phi(\mathbf{n})$ can be easily calculated and are given by

$$
\begin{aligned}
& H_{0} \Phi(\mathbf{n})=\left(\sum_{j=1}^{N} n_{j}^{2}\right) \Phi(\mathbf{n}) \\
& H_{1} \Phi(\mathbf{n})=\sum_{j<k}\left(n_{j}-n_{k}\right)\left(\Phi(\mathbf{n})+2 \sum_{s=1}^{n_{j}-n_{k}-1} \Phi\left(\ldots, n_{j}-s, \ldots, n_{k}+s, \ldots\right)\right) .
\end{aligned}
$$

$H_{0}$ generates only the basis state $\Phi(\mathbf{n})$ itself while $H_{1}$ is responsible for generating a family of states $\Phi\left(\ldots, n_{j}-s, \ldots, n_{k}+s, \ldots\right)$ which are obtained from $\Phi(\mathbf{n})$ by all possible pairwise 
"squeezing" of the quantum numbers. If a state is generated from another by squeezing a pair of quantum numbers by one unit (i.e., $n_{j} \rightarrow n_{j}-1, n_{k} \rightarrow n_{k}+1$ for $n_{j}-n_{k} \geq 2$ ), then I call the former "daughter-state" and the latter its "mother-state."

The family of states can be organized into levels such that the members of a given level are mutually not related or unreachable (i.e., no mother-daughter relationship exists between the members in the same level) and the daughters of a member from a given level always belong to a lower level in the family. The highest-level mother-state denoted by $|\nu\rangle_{1}$, where $\nu$ is a level index and is equal to the total number of levels in the family, generates the entire family of daughter-states which are denoted by $|\mu\rangle_{\alpha}$ where $1 \leq \mu<\nu$ and $\alpha$ an index for the states in the $\mu$ th level. While the order of the levels in the family can be uniquely determined, the order within a level is quite arbitrary.

To provide an illustration of the above mentioned family structure, I give the following example. Let the highest-level mother-state be $|6\rangle_{1}=\Phi(4,3,1,0)$. Then, the members of the family are $|5\rangle_{1}=\Phi(4,2,2,0),|4\rangle_{1}=\Phi(4,2,1,1),|4\rangle_{2}=\Phi(3,3,2,0),|3\rangle_{1}=\Phi(3,3,1,1)$, $|2\rangle_{1}=\Phi(3,2,2,1)$, and $|1\rangle_{1}=\Phi(2,2,2,2)$. A pictorial representation of the family structure is shown in Fig. 1 and I call it a level diagram. In Fig. 1 the states are represented by dots and each arrow connects a state $A$ to $B$ where $B$ is reachable from $A$ by squeezing on a pair of quantum numbers for $A$ by one unit (i.e., connects a mother and her daughter). The levels are ordered from top to bottom, from the most squeezable to unsqueezable states, such that the highest-level mother-state is at the top; and therefore the arrows always point downwards and never upwards. A set of arrows that is topologically equivalent to a directed line forms a path. A weight $\mathcal{W}$ of an arrow is defined to be $m\left(n_{i}\right) m\left(n_{j}\right)$, where $n_{i}$ and $n_{j}$ are the two quantum numbers squeezed to produce a daughter and $m(l)$ the multiplicity of $l$ in the quantum number set specifying the mother-state. In Fig. 1 the numbers next to the arrows are the corresponding weights. The states in the same level are not connected; hence, the Hamiltonian is diagonal in that subspace.

A subfamily of a family can also be constructed by starting from a given state, which would be the highest-level mother-state of that subfamily, and grouping all her reachable 
off-springs. The total number of subfamilies is therefore equal to the dimension (i.e., the total number of basis states) of the family.

The lowest-level states (the unsqueezable states) clearly have to be of one of the two types: (I) $\Phi(\ldots, m, m, m, m, m, \ldots)$ or (II) $\Phi(\ldots, m, m, m, m, m-1, m-1, m-1, m-1, \ldots)$. The type-I state corresponds to the ground state up to a global Galilean boost and the typeII to states with a single hole excitation. These one-hole states are eigenstates of $\tilde{H}$ and, furthermore, since the following superposed state

$$
\Psi(x)=\prod_{j=1}^{N}\left(z-z_{j}\right),
$$

where $z=\exp (i 2 \pi x / L)$, can be expanded purely in terms of the one-hole eigenstates, $\Psi(x)$ describes a state with a hole localized at $x$.

The matrix representation of $\tilde{H}$ acting on the partially ordered state space is always triangular since the action of $\tilde{H}$ on a given state always generates states belonging to lower levels. The eigenvalues, therefore, are simply given by the diagonal matrix elements. In particular the energy of an eigenstate spanned by a family with the highest-level motherstate $\Phi(\mathbf{n})$ is given by

$$
E_{\mathbf{n}}^{(0)}=\sum_{j=1}^{N} n_{j}^{2}+\lambda \sum_{j<k}\left(n_{j}-n_{k}\right)
$$

The off-diagonal elements are given by

$$
{ }_{\alpha}\langle\mu|\tilde{H}| \nu\rangle_{\beta}= \begin{cases}{\left[\sum_{\mathcal{P}}\left(\prod_{i \in \mathcal{P}} \mathcal{W}_{i}\right)\right] E_{\mathbf{n}}^{(1)}} & \text { if } \nu>\mu \\ 0 & \text { if } \nu \leq \mu\end{cases}
$$

where $E_{\mathbf{n}}^{(1)}=2 \lambda \sum_{j<k}\left(n_{j}-n_{k}\right)$, and the sum is over all possible path $\mathcal{P}$ from $|\nu\rangle_{\beta}$ to $|\mu\rangle_{\alpha}$ and the product over all the weights $\mathcal{W}_{i}$ of the intermediate arrows belonging to $\mathcal{P}$.

For the example given in Fig. 1, $\tilde{H}$ is represented by 


$$
\tilde{H}=\left(\begin{array}{ccccccc}
\varepsilon_{(1,1)}^{(1,1)} & \varepsilon_{(2,1)}^{(1,1)} & \varepsilon_{(3,1)}^{(1,1)} & \varepsilon_{(4,1)}^{(1,1)} & \varepsilon_{(4,2)}^{(1,1)} & \varepsilon_{(5,1)}^{(1,1)} & \varepsilon_{(6,1)}^{(1,1)} \\
0 & \varepsilon_{(2,1)}^{(2,1)} & \varepsilon_{(3,1)}^{(2,1)} & \varepsilon_{(4,1)}^{(2,1)} & \varepsilon_{(4,2)}^{(2,1)} & \varepsilon_{(5,1)}^{(2,1)} & \varepsilon_{(6,1)}^{(2,1)} \\
0 & 0 & \varepsilon_{(3,1)}^{(3,1)} & \varepsilon_{(4,1)}^{(3,1)} & \varepsilon_{(4,2)}^{(3,1)} & \varepsilon_{(5,1)}^{(3,1)} & \varepsilon_{(6,1)}^{(3,1)} \\
0 & 0 & 0 & \varepsilon_{(4,1)}^{(4,1)} & 0 & \varepsilon_{(5,1)}^{(4,1)} & \varepsilon_{(6,1)}^{(4,1)} \\
0 & 0 & 0 & 0 & \varepsilon_{(4,2)}^{(4,2)} & \varepsilon_{(5,1)}^{(4,2)} & \varepsilon_{(6,1)}^{(4,2)} \\
0 & 0 & 0 & 0 & 0 & \varepsilon_{(5,1)}^{(5,1)} & \varepsilon_{(6,1)}^{(5,1)} \\
0 & 0 & 0 & 0 & 0 & 0 & \varepsilon_{(6,1)}^{(6,1)}
\end{array}\right),
$$

where $\varepsilon_{(\nu, \beta)}^{(\mu, \alpha)}={ }_{\alpha}\langle\mu|\tilde{H}| \nu\rangle_{\beta}$. For example, $\varepsilon_{(6,1)}^{(3,1)}=7 \times E_{(3,3,1,1)}^{(1)}$ since there are five different ways to get from $|6\rangle_{1}$ to $|3\rangle_{1}$ with the following corresponding weights (see Fig. 1): (a) $|6\rangle_{1} \stackrel{1}{\rightarrow}|4\rangle_{1} \stackrel{1}{\rightarrow}|3\rangle_{1} ;(\mathrm{b})|6\rangle_{1} \stackrel{1}{\rightarrow}|5\rangle_{1} \stackrel{2}{\rightarrow}|4\rangle_{1} \stackrel{1}{\rightarrow}|3\rangle_{1} ;(\mathrm{c})|6\rangle_{1} \stackrel{1}{\rightarrow}|3\rangle_{1} ;(\mathrm{d})|6\rangle_{1} \stackrel{1}{\rightarrow}|4\rangle_{2} \stackrel{1}{\rightarrow}|3\rangle_{1} ;(\mathrm{e})$ $|6\rangle_{1} \stackrel{1}{\rightarrow}|5\rangle_{1} \stackrel{2}{\rightarrow}|4\rangle_{2} \stackrel{1}{\rightarrow}|3\rangle_{1}$.

The eigenenergy given by Eq. (2.10) plus the ground state energy can be rewritten in terms of newly defined pseudomomenta $k_{j}$ as

$$
E=\frac{\hbar^{2}}{2 m} \sum_{j=1}^{N} k_{j}^{2}
$$

where

$$
L k_{j}=2 \pi I_{j}+\pi(\lambda-1) \sum_{l=1}^{N} \operatorname{sgn}\left(k_{j}-k_{l}\right) .
$$

The quantum numbers $I_{j}$ are now distinct (half-odd) integers and are related to $n_{j}$ 's by $I_{j}=n_{j}+(N+1-2 j) / 2$.

The distribution of $k_{j}$ determined by Eq. (2.14) is used to construct pictorial representations of the eigenstates called motifs which are crucial for exposing the fractional statistics obeyed by the elementary excitations of the model. Detailed discussion of this subject is given in Section IV.

\section{JACK SYMMETRIC POLYNOMIALS}

The polynomial solutions of Eq. (2.3) in Section III is also known in mathematical literature as Jack polynomials 25]. In fact, Stanley [26] has shown that the complete set of 
linearly independent solutions of Eq. (2.3) is indeed given by Jack polynomials up to global Galilean boosts (i.e., up to the factor $\prod_{j=1}^{N} z_{j}^{J}$ where $J$ is the current and takes an arbitrary real number) [6].

For better readability this section is divided into two subsections: the first introduces the conventional notations used in mathematical literatures and the second some of the general properties of Jack polynomials.

\section{A. Introduction to notations}

Partitions are defined as sequences of non-negative integers in non-increasing order and are used to label the symmetric polynomials. They are denoted by bold-face Greek letters as

$$
\boldsymbol{\kappa}=\left(\kappa_{1}, \kappa_{2}, \ldots, \kappa_{N}\right)
$$

where $\kappa_{1} \geq \kappa_{2} \geq \ldots \geq \kappa_{N}$. Non-zero $\kappa_{j}$ are called parts of $\boldsymbol{\kappa}$ whose length (i.e., the total number of non-zero parts) is denoted by $\ell(\boldsymbol{\kappa})$. The weight of the partition is defined by $|\boldsymbol{\kappa}|=\sum_{j=1}^{\ell(\boldsymbol{\kappa})} \kappa_{j}$. If $\kappa_{1}+\ldots+\kappa_{i} \geq \mu_{1}+\ldots+\mu_{i}$ for all $i \geq 1$, then $\boldsymbol{\kappa} \geq \boldsymbol{\mu}$.

Young diagram $\mathcal{D}(\boldsymbol{\kappa})$ is used to graphically represent a partition: $\mathcal{D}(\boldsymbol{\kappa})=\{(i, j): 1 \leq$ $\left.i \leq \ell(\boldsymbol{\kappa}), 1 \leq j \leq \kappa_{i}\right\}$. The cell labeled by $(i, j)$ is situated in the $i$-th row and the $j$-th column of the Young diagram. The diagram of $\boldsymbol{\kappa}$, therefore, consists of $\ell(\boldsymbol{\kappa})$ rows of lengths $\kappa_{j}$.

A conjugate of $\boldsymbol{\kappa}$ is denoted by $\boldsymbol{\kappa}^{\prime}=\left(\kappa_{1}^{\prime}, \kappa_{2}^{\prime}, \ldots\right)$ and corresponds to a partition whose diagram is obtained by changing all the rows of $\mathcal{D}(\boldsymbol{\kappa})$ to columns in non-increasing order from the left to right. For example, the conjugate of $\boldsymbol{\kappa}=(5,2,2,1)$ is $\boldsymbol{\kappa}^{\prime}=(4,3,1,1,1)$. Now, the following simple but useful identity can be derived 27]

$$
n(\boldsymbol{\kappa}) \equiv \sum_{i=1}^{\ell(\boldsymbol{\kappa})}(i-1) \kappa_{i}=\sum_{i=1}^{\ell\left(\boldsymbol{\kappa}^{\prime}\right)}\left(\begin{array}{c}
\kappa_{i}^{\prime} \\
2
\end{array}\right) .
$$

In order prove Eq. (3.2) every cell in the $i$ th row of $\mathcal{D}(\boldsymbol{\kappa})$ is filled in with an integer $i-1$. Since $n(\boldsymbol{\kappa})$ corresponds to the sum of all the integers in the diagram, the two different 
expressions for $n(\boldsymbol{\kappa})$ are obtained depending on whether the numbers in each row or column are summed first.

For a given cell $s=(i, j)$ of a diagram $\mathcal{D}(\boldsymbol{\kappa})$ there are corresponding arm-length $a(s)=$ $\kappa_{i}-j$, arm-colength $a^{\prime}(s)=j-1$, leg-length $l(s)=\kappa_{j}^{\prime}-i$, and leg-colength $l^{\prime}(s)=i-1$. The upper and lower hook-lengths are defined, respectively, as

$$
\begin{aligned}
& h_{\boldsymbol{\kappa}}^{*}(s)=l(s)+\frac{1+a(s)}{\lambda}, \\
& h_{*}^{\boldsymbol{\kappa}}(s)=l(s)+1+\frac{a(s)}{\lambda} .
\end{aligned}
$$

\section{B. General properties of Jack polynomials}

The symmetric polynomials are indexed by the partitions. The bosonic basis functions $\Phi(\mathbf{n})$ of the CSM are called the monomial symmetric functions and the quantum numbers $\mathbf{n}$ correspond to the partitions defined in the previous subsection. Since the quantum numbers are allowed to be negative integers, the correspondence is only up to some trivial constant translation or the global Galilean boost. This restriction to non-negative integer parts is more of a convenience than a restriction since the CSM Hamiltonian is invariant under the global Galilean transformation.

I shall denote Jack symmetric polynomials as $J_{\kappa}^{1 / \lambda}\left(z_{1}, \ldots, z_{N}\right)$ which are the solutions of Eq. (2.3). If $\lambda=1$, Jack polynomials reduce to Shur functions which describe the excited states of the free fermions. At $\lambda=0$, it becomes the monomial symmetric function which is just the free bosonic wavefunction. If $\lambda=2$ or $1 / 2$, they are called the zonal spherical functions. As $\lambda \rightarrow \infty, J_{\kappa}^{1 / \lambda}$ reduce to the elementary symmetric functions.

One way of defining Jack polynomials is through the differential equation (2.3). The other is based on the properties of the power-sum symmetric function $p_{\boldsymbol{\kappa}}=p_{\kappa_{1}} p_{\kappa_{2}} p_{\kappa_{3}} \ldots$, where $p_{\kappa_{\nu}}=\sum_{j} z_{j}^{\kappa_{\nu}}$. Define a bilinear scalar product on the vector space of all symmetric functions of finite degree as

$$
\left\langle p_{\boldsymbol{\kappa}}, p \boldsymbol{\mu}\right\rangle_{1 / \lambda}=\delta_{\boldsymbol{\kappa}, \boldsymbol{\mu}} z \boldsymbol{\kappa} \lambda^{-\ell(\boldsymbol{\kappa})}
$$


where $z_{\boldsymbol{\kappa}}=\prod_{i \geq 1} i^{m_{i}} m_{i}$ !, and $m_{i}=m_{i}(\boldsymbol{\kappa})$ is the number of parts of $\boldsymbol{\kappa}$ equal to $i$. Using this definition, Macdonald [28] proved that there are unique symmetric functions satisfying the following three properties:

1. Orthogonality: $\left\langle J_{\boldsymbol{\kappa}}, J_{\boldsymbol{\mu}}\right\rangle_{1 / \lambda}=\delta_{\boldsymbol{\kappa}, \boldsymbol{\mu}} j_{\boldsymbol{\kappa}}^{\lambda}$, where $j_{\boldsymbol{\kappa}}^{\lambda}$ is the normalization constant.

2. Triangularity: $J_{\boldsymbol{\kappa}}=\sum_{\boldsymbol{\mu}} v_{\boldsymbol{\kappa} \boldsymbol{\mu}} \Phi(\boldsymbol{\mu})$, where $v_{\boldsymbol{\kappa} \boldsymbol{\mu}}=0$ unless $\boldsymbol{\kappa} \leq \boldsymbol{\mu}$.

3. Normalization: If $|\boldsymbol{\kappa}|=n$, then $v_{\boldsymbol{\kappa} \boldsymbol{\mu}}=n$ !, where $\boldsymbol{\kappa}=(\underbrace{1,1, \ldots, 1}_{n})$.

$J_{\boldsymbol{\kappa}}$ are, then, constructed by Gram-Schmidt orthogonalization relative to the scalar product on the ring of polynomials. Stanley [26] proved that the normalization constant is given by

$$
j_{\boldsymbol{\kappa}}^{\lambda}=\prod_{s \in \boldsymbol{\kappa}} h_{\boldsymbol{\kappa}}^{*}(s) h_{*}^{\boldsymbol{\kappa}}(s)
$$

There is another scalar product on which Jack polynomials are orthogonal:

$$
\begin{aligned}
\langle\boldsymbol{\kappa} \mid \boldsymbol{\mu}\rangle_{1 / \lambda} & \equiv A_{N}^{2}\left(\prod_{j=1}^{N} \int_{0}^{L} d x_{j}\right) \overline{J_{\boldsymbol{\kappa}}^{1 / \lambda}\left(z_{1}, z_{2}, \ldots, z_{N}\right)} J_{\boldsymbol{\mu}}^{1 / \lambda}\left(z_{1}, z_{2}, \ldots, z_{N}\right) \prod_{i<j}\left|z_{i}-z_{j}\right|^{2 \lambda} \\
& =A_{N}^{2} j_{\boldsymbol{\kappa}}^{\lambda} \prod_{s \in \boldsymbol{\kappa}} \frac{N+a^{\prime}(s) / \lambda-l^{\prime}(s)}{N+\left(a^{\prime}(s)+1\right) / \lambda-\left(l^{\prime}(s)+1\right)} \delta_{\boldsymbol{\kappa}, \boldsymbol{\mu}}
\end{aligned}
$$

where $z_{j}=\exp \left(i 2 \pi x_{j} / L\right)$ and $A_{N}^{2}=(1 / L)^{N} \Gamma^{N}(1+\lambda) / \Gamma(1+\lambda N)$, and the bar over the polynomial denotes the complex conjugation. Note also that the multidimensional integral above is equal to $L^{N}$ times the constant term in

$$
J_{\kappa}^{1 / \lambda}\left(1 / z_{1}, 1 / z_{2}, \ldots, 1 / z_{N}\right) J_{\mu}^{1 / \lambda}\left(z_{1}, z_{2}, \ldots, z_{N}\right) \prod_{i \neq j}\left(1-\frac{z_{i}}{z_{j}}\right)^{\lambda}
$$

Eq. (3.7) has been conjectured first by Macdonald [29] and then later proved by himself 28] and also by Kadell [30].

Since Jack polynomials span the vector space of symmetric functions, they can be used to expand any symmetric functions. This property is particularly useful in calculating the correlation functions of the CSM as will be shown later in this paper. Here are some of them 31,32: 


$$
\begin{array}{r}
\sum_{i=1}^{N} z_{i}^{n}=\frac{n}{\lambda} \sum_{|\boldsymbol{\kappa}|=n} \frac{\left[0^{\prime}\right]_{\boldsymbol{\kappa}}^{\lambda}}{j_{\boldsymbol{\kappa}}^{\lambda}} J_{\boldsymbol{\kappa}}^{1 / \lambda}\left(z_{1}, \ldots, z_{N}\right), \\
\prod_{j=1}^{N}\left(1-z_{j}\right)^{a}=\sum_{\boldsymbol{\kappa}} \frac{\{-a\}_{\boldsymbol{\kappa}}^{\lambda}}{\lambda^{|\boldsymbol{\kappa}|} j_{\boldsymbol{\kappa}}^{\lambda}} J_{\boldsymbol{\kappa}}^{1 / \lambda}\left(z_{1}, \ldots, z_{N}\right),
\end{array}
$$

where $[a]_{\boldsymbol{\kappa}}^{\lambda}=\prod_{(i, j) \in \boldsymbol{\kappa}}\{a+(j-1) / \lambda-(i-1)\}$, and $\{a\}_{\boldsymbol{\kappa}}^{\lambda}=\prod_{(i, j) \in \boldsymbol{\kappa}}\{a-\lambda(i-1)+(j-1)\}$. The sum in Eq. (3.10) extends over all possible partitions while in Eq. (3.9) it is restricted to partitions with weight $|\boldsymbol{\kappa}|=n$. The prime in $\left[0^{\prime}\right]_{\boldsymbol{\kappa}}^{\lambda}$ denotes that the product does not include the cell $(0,0)$; otherwise the total product is trivially equal to zero. $J_{\boldsymbol{\kappa}}$ also satisfies

$$
J_{\boldsymbol{\kappa}}^{1 / \lambda}\left(w z_{1}, w z_{2}, \ldots, w z_{N}\right)=w^{|\boldsymbol{\kappa}|} J_{\boldsymbol{\kappa}}^{1 / \lambda}\left(z_{1}, z_{2}, \ldots, z_{N}\right)
$$

since Jack polynomials are homogeneous functions of degree $|\boldsymbol{\kappa}|$.

\section{FRACTIONAL STATISTICS}

I divide this section into two subsections. In the first (second) subsection the fractional exchange (exclusion) statistics is discussed in the context of one-dimensional models. The exchange statistics in two-dimension is directly relevant to the fractional quantum Hall effect and various people have made contributions to this fascinating field [33]. In one-dimension, however, the definition of fractional exchange statistics is rather obscure and incomplete with a possible exception of the CSM. One the other hand, the definition of fractional exclusion statistics is spatial dimension independent and is based on the structure of the Hilbert space rather than the configuration space. While the fermions obey the well-known Pauli exclusion principle, more exotic particles may obey a "generalized exclusion principle." [9]

\section{A. Exchange Statistics}

The first full mathematical treatment of the fractional statistics is due mainly to Leinaas and Myrheim who used the multiply connected topological structure of the configuration 
space of collections of identical particles to show the possibility of exotic statistics in spatial dimension less than three [2]. While their idea has been extensively applied to twodimensional systems especially in the context of the fractional quantum Hall effect, very little attention has been paid to the one-dimensional (1D) systems. Perhaps the main difficulty in 1D systems is that the physical exchanges of the particles necessarily involve scattering processes and that there is no known unique way of un-tangling the kinematic aspect of the fractional statistics from the dynamical processes.

For integrable 1D quantum systems, however, there is a general consistency condition known as the Yang-Baxter equation (YBE) which essentially puts strong restrictions on the scattering matrices. Therefore, intuitively the braiding of the particle "trajectories" in onedimension when properly defined may be given by the YBE [34]. In fact the YBE is known to be intimately related to the the knot theory and the braid groups [35]. Hence, perhaps the following quantum Yang-Baxter equation should be interpreted as the one-dimensional generalized braiding relations,

$$
S_{12}(v-u) S_{13}(v) S_{23}(u)=S_{23}(u) S_{13}(v) S_{12}(v-u)
$$

where $S_{i j}(v)$ is the scattering matrix in the tensor product of linear vector spaces, $V \otimes V \otimes V$ and acts non-trivially only in the $i$ th and $j$ th space, e.g. $S_{12}(v)=R(v) \otimes I$ where $R(v)$ is a matrix defined in $V \otimes V$. The parameters $v$ and $u$ are called spectral parameters and are equal to the rapidity differences between two colliding particles. One can rewrite Eq. (4.1) in terms of $\tilde{R}(v)=P R(v)$ where $P$ denotes the transposition, $P x \otimes y=y \otimes x$. Define matrices $\tilde{R}_{i}(v)=I \otimes \cdots \otimes \tilde{R}(v) \otimes \cdots \otimes I$ on $V \otimes \cdots \otimes V$, where $\tilde{R}(v)$ acts on $i$-th and $i+1$-th spaces. The matrices $\tilde{R}_{i}(v)$ satisfy the following relations

$$
\begin{aligned}
\tilde{R}_{i}(v) \tilde{R}_{j}(u) & =\tilde{R}_{j}(u) \tilde{R}_{i}(v) \quad \text { if }|i-j| \geq 2, \\
\tilde{R}_{i+1}(v-u) \tilde{R}_{i}(v) \tilde{R}_{i+1}(u) & =\tilde{R}_{i}(u) \tilde{R}_{i+1}(v) \tilde{R}_{i}(v-u) .
\end{aligned}
$$

Without the spectral parameters the $\tilde{R}$ matrices satisfy the braiding relations. The complete description of the fractional statistics based on the YBE needs to be worked out. 
At present it seems unclear to me how the construction based on the YBE can be applied to a general 1D (i.e., not necessarily integrable) systems. Maybe there is a way to map a class of general 1D models to integrable models with in some sense small residual interactions that break the integrability. Instead of pursuing this incomplete Yang-Baxter story any further, I give here somewhat heuristic but intuitive device for constructing the 1D fractional statistics. The $N$-particle state is constructed as follow

$$
\int d x_{1} \cdots d x_{N} \Psi\left(x_{1}, \ldots, x_{N}, t\right) \phi^{*}\left(x_{N}, t\right) \cdots \phi^{*}\left(x_{1}, t\right)|0\rangle
$$

where $\phi^{*}(x, t)$ is the anyon creation operator. The "anyon" fields satisfy the following equal time commutation relations

$$
[\phi(x, t), \phi(y, t)]_{\lambda}=\left[\phi^{*}(x, t), \phi^{*}(y, t)\right]_{\lambda}=\left[\phi(x, t), \phi^{*}(y, t)\right]_{\lambda}=0 \text { if } x \neq y,
$$

where $[x, y]_{\lambda}=x y+\exp (i \pi \lambda \operatorname{sgn}(y-x)) y x$.

The wavefunction $\Psi\left(x_{1}, \ldots, x_{N}, t\right)$ is multi-valued and satisfies

$$
\Psi\left(\ldots, x_{i}, \ldots, x_{j} \ldots, t\right)=e^{i \pi \lambda \operatorname{sgn}\left(x_{i}-x_{j}\right)} \Psi\left(\ldots, x_{j}, \ldots, x_{i}, \ldots, t\right)
$$

The integrand in Eq. (4.4), however, is always single valued since the phases arising from the wavefunction and the "anyon" field operators are set to cancel each other.

If I specialize to the CSM the fractional exchange statistics can be formulated in the first quantized language. First, the Hamiltonian (2.1) can be rewritten as [36]

$$
H=\frac{1}{2 m} \sum_{j=1}^{N}\left(p_{j}+i \frac{\pi \hbar \lambda}{L} \sum_{k(\neq j)} \cot \left[\frac{\pi\left(x_{j}-x_{k}\right)}{L}\right] P_{j k}\right)^{2},
$$

where $p_{j}=-i \hbar \partial_{x_{j}}$ is the momentum operator and $P_{i j}$ the particle exchange operator. The extra term added to the momentum operator is an 1D analog of the Chern-Simons gauge field.

In two-dimension there are two well-known ways to code the fractional statistics for the ideal anyon gas. One is to take the free Hamiltonian and require that its wavefunctions be multi-valued as in Eq. (4.6). The other is to introduce the Chern-Simons gauge field and 
write the Hamiltonian in terms of this gauge field and further require that the wavefunctions be single valued and symmetric. The Hamiltonian (4.7) corresponds to the $1 \mathrm{D}$ version of the second formulation of the fractional exchange statistics. Hence, the following symmetric wavefunctions are the eigenstates of the 1D anyon system

$$
\Psi_{\boldsymbol{\kappa}}^{\lambda}\left(x_{1}, \ldots, x_{N}\right)=\varphi\left(x_{1}, \ldots, x_{N}\right) \Psi_{0} J_{\boldsymbol{\kappa}}^{\lambda}
$$

where the "ordering function" $\varphi$ is introduced to maintain the total wavefunction symmetric [6]. In particular $\varphi$ keeps track of the braiding of the particles and is set to cancel the exchange phases arising from $\Psi_{0}$. I require the ordering function to satisfy

$$
\varphi\left(x_{1}, \ldots, x_{m}, \ldots, x_{N}\right)=e^{i(m-1) \pi \lambda} \varphi\left(x_{1}, \ldots, \hat{x}_{m}, \ldots, x_{N}\right),
$$

where the hat over the variable denotes the absence of that variable from the function. In other words, to remove the particle at $x_{m}$ it is necessary to pass through $m-1$ particles.

In Section VIT I construct explicit second quantized "anyon" fields and show their consistency with the first quantized formulation of the fractional exchange statistics for the CSM.

\section{B. Exclusion Statistics}

The notion of fractional exclusion statistics based on the so called "generalized Pauli exclusion principle" has first been formulated by Haldane and applied to the elementary topological excitations of general condensed matter systems [9]. This new concept of statistics is based on the structure of the single particle Hilbert space of the elementary excitations. More specifically, the change in the size of the available states $(\Delta D)$ in the Hilbert space as the number of particles (i.e., the elementary excitations) is changed $(\Delta N)$ for a given system with fixed boundary condition defines the statistics of the particles with the statistical parameter defined as $g=-\Delta D / \Delta N$. Hence, for example, the bosons and fermions are identified with $g=0$ and $g=1$, respectively. 
In order to facilitate proper understanding of the exclusion statistics in the context of the CSM I introduce a pictorial representation of the eigenstates and make the identification of the excitation contents of the states easier [6]. Eq. (2.14) gives the occupation configurations of the pseudomomenta $k_{j}$ for all the eigenstates of the CSM. The quantum numbers $\left\{I_{j}\right\}$ in Eq. (2.14) are distinct (half-odd) integers and in the ground state are given by the following set

$$
\left\{I_{j}^{0}\right\}=\left\{-\frac{N-1}{2},-\frac{N-3}{2}, \ldots, \frac{N-3}{2}, \frac{N-1}{2}\right\}
$$

Therefore, the ground state pseudomomenta are given by

$$
\left\{k_{j}^{0}\right\}=\left\{-\frac{\pi \lambda}{L}(N-1),-\frac{\pi \lambda}{L}(N-3), \ldots, \frac{\pi \lambda}{L}(N-3), \frac{\pi \lambda}{L}(N-1)\right\} .
$$

The total ground state energy $E^{0}=\sum_{j}\left(k_{j}^{0}\right)^{2}$ is equal to $\pi^{2} \lambda^{2} N\left(N^{2}-1\right) / 3 L^{2}$. The excited states are given by integer displacements of $\left\{I_{j}^{0}\right\}$. Therefore, two neighboring pseudomomenta for any arbitrary state must be separated by

$$
\Delta k_{j} \equiv\left|k_{j}-k_{j-1}\right|=\frac{2 \pi}{L}(\lambda+l)
$$

where $l$ is a non-negative integer.

In order to construct a picture that exposes the excitation content of the excited states, I let $\lambda$ to be a rational number $p / q$ with $p$ and $q$ coprimes and introduce one-dimensional lattice with the lattice spacing equal to $2 \pi / q L$. I assign each lattice point with 1 if that lattice point coincides with the value of one of the occupied pseudomomenta and with 0 if it does not. Hence, the ground state for $\lambda=3 / 2$ and $N=10$ is represented by ...0000000010010010010010010010010010010000000 ... All the other excited states can be obtained from this ground state configuration by displacing the ones such that the number of zeroes between any pair of ones is equal to $p-1+q l$ where $l$ is a non-negative integer.

I use three different names for the particles in the model-real, pseudo, and quasiparticles. The real particles are, of course, the physical quantum particles described by the canonically conjugate coordinate and momentum variables $\left\{x_{j}, p_{j}\right\}$. The pseudoparticles 
are described by the pseudomomentum operator (see Eq. (4.7)) which a sum of the usual momentum and the 1D "statistical gauge field." The quasiparticles are the elementary excitations of the system. Because the pseudoparticles form an ideal gas, the quasiparticles are essentially same as the pseudoparticles excited out of the condensate. The holes left behind in the pseudoparticle condensate will be called quasiholes. Hence, the name "pseudo" and "quasi" will be used interchangeably in some cases [6].

Since $p-1$ zeroes are always required between the ones I call them bound zeroes which seem to represent the mutual statistical exclusions. In the case of the free Fermions carrying the flux $2 \pi$ in units where $e=\hbar=c=1$ so that the flux quanta $\Phi_{0}=2 \pi$, the minimum separation of the momenta is $2 \pi / L$ which can be considered as the mutual Pauli exclusion. In this case the minimum separation is $2 \pi \lambda$; therefore, it is natural to assign $2 \pi \lambda$ flux attached to the pseudoparticles. The remaining zeroes are called unbound zeroes. The $q$ consecutive unbound zeroes in the condensate of the pseudoparticles constitute a single hole excitation. Thus, if a pseudoparticle is removed from the ground state condensate, then there are $p$ unbound zeroes in place where the one is removed. This state is forbidden if $q \neq 1$. In general a minimum of $q$ ones must be removed so that they leave behind at least $p q$ unbound zeroes which break up into $p$ holes. From the view point of the particles (holes) the change in the number of available single particle states is $p(-q)$ while the change in the number of quasiparticles (quasiholes) in the system is $-q(p)$. Therefore, the statistical parameter $g$ for the quasiparticle (quasihole) is $g=p / q=\lambda(g=q / p=1 / \lambda)$. This inverse relationship between the statistical parameters of the particles and holes is essentially the Chern-Simons duality. To summarize the fractional exclusion statistics, q particle excitations are accompanied by $p$ hole excitations.

The configurations constructed above are representations of the diagrams of partitions $\mathcal{D}(\boldsymbol{\kappa})$ introduced in Sec. III]. The part $\kappa_{j}$ corresponds to the displacement of $j$ th quantum number from the ground state (i.e., $I_{j}-I_{j}^{0}$ if $I_{1}>I_{2}>\ldots>I_{N}$ ). The excitations given by $\boldsymbol{\kappa}$ include only the states with non-negative displacements (i.e., $k_{j}$ moved only to the right) and all the other states are obtained by global Galilean transformations. Therefore, each 
row (column) in the diagram corresponds to the particle (hole) excitations of the CSM.

Following Yang and Yang [37] and Sutherland [22] I can also construct the thermodynamics [6]. In the thermodynamic limit it is convenient to define the hole distribution $\rho_{h}(k)$ and the particle distribution function $\rho_{p}(k)$. The function $\rho_{p}(k)$ are given by the solutions of Eq. (2.14) while $\rho_{h}(k)$ by the corresponding complementary equations given by the unused quantum numbers $I_{j}$; thus, they satisfy $1=\rho_{h}(k)+\lambda \rho_{p}(k)$. This equation states that one hole and $1 / \lambda$ particles (or $\lambda$ holes and one particle) have equal weight in occupying the volume in $k$-space. Hence, for $\lambda \neq 1$ the particle-hole symmetry is broken; and in the bosonic case $(\lambda=0)$ the symmetry is maximally broken. With a proper normalization one can equally state that $1=(1 / \lambda) \rho_{h}(k)+\rho_{p}(k)$. This particle-hole duality is the essence of the Chern-Simons duality.

The thermodynamic function is given by $\Omega=E-T S-\mu N$, where the energy $(E)$, the particle number $(\mathrm{N})$, and the entropy $(S)$ are given by

$$
\begin{aligned}
& E=V \int d k \rho_{p}(k) e(k), \\
& N=V \int d k \rho_{p}(k), \\
& S=V \int d k\left\{\left(\rho_{h}+\rho_{p}\right) \log \left(\rho_{h}+\rho_{p}\right)-\rho_{h} \log \rho_{h}-\rho_{p} \log \rho_{p}\right\} .
\end{aligned}
$$

Here, $V$ is the volume of the system. By minimizing $\Omega\left(\rho_{p}, \rho_{h}\right)$ with respect to the density functions the following relation can easily be obtained

$$
\left(1-\lambda \rho_{p}(k)\right)^{\lambda}\left(1+(1-\lambda) \rho_{p}(k)\right)^{1-\lambda}=\rho_{p}(k) e^{(e(k)-\mu) / T} .
$$

This equation is also obtained by $\mathrm{Wu}$ using a different method [39].

\section{DYNAMICAL DENSITY-DENSITY CORRELATION FUNCTION}

In this section I show that the dynamical density-density correlation function (DDDCF) can be calculated exactly for the CSM using the known properties of Jack polynomials. The DDDCF is defined by 


$$
\begin{aligned}
{ }_{N}\left\langle 0\left|\rho\left(x^{\prime}, t^{\prime}\right) \rho(x, t)\right| 0\right\rangle_{N} & ={ }_{N}\left\langle 0\left|e^{i H_{N} t^{\prime}} \rho\left(x^{\prime}\right) e^{-i H_{N} t^{\prime}} e^{i H_{N} t} \rho(x) e^{-i H_{N} t}\right| 0\right\rangle_{N} \\
& ={ }_{N}\left\langle 0\left|\rho\left(x^{\prime}\right) e^{-i\left(H_{N}-E_{N}^{0}\right)\left(t^{\prime}-t\right)} \rho(x)\right| 0\right\rangle_{N},
\end{aligned}
$$

where the reduced density operator $\rho(x)=(1 / L) \sum_{j} \delta\left(x-x_{j}\right)-N / L$ and $|0\rangle_{N}$ the normalized $N$-particle ground state.

The first step in calculating the DDDCF is to expand $\rho(x)|0\rangle_{N}$ in terms of the eigenstates of the CSM (i.e., Jack polynomials). The delta function $\delta(x)$ is a periodic function with a period $L$ and thus can be expressed as a Fourier sum $(1 / L) \sum_{m=-\infty}^{+\infty} \exp (i 2 \pi x m / L)$. Therefore, I can write $\rho(x)$ as follow

$$
\rho(x)=\frac{1}{L} \sum_{m=1}^{\infty}\left(z^{m} p_{-m}+z^{-m} p_{m}\right),
$$

where $z=\exp (i 2 \pi x / L), z_{j}=\exp \left(i 2 \pi x_{j} / L\right)$, and $p_{m}=\sum_{j=1}^{N} z_{j}^{m}$. The power sum $p_{m}$ can be expanded in terms of Jack polynomials using the identity Eq. (3.9).

Using the orthogonality relation Eq. (3.7) and its extension Eq. (3.8), I obtain the following expression for the DDDCF

$$
{ }_{N}\langle 0|\rho(x, t) \rho(0,0)| 0\rangle_{N}=\frac{1}{L^{2}} \frac{2}{\lambda^{2}} \sum_{\boldsymbol{\kappa}} \frac{|\boldsymbol{\kappa}|^{2}}{j_{\boldsymbol{\kappa}}^{\lambda}} \frac{\left(\left[0^{\prime}\right]_{\boldsymbol{\kappa}}^{\lambda}\right)^{2}[N]_{\boldsymbol{\kappa}}^{\lambda}}{[N+1 / \lambda-1]_{\boldsymbol{\kappa}}^{\lambda}} \cos (2 \pi|\boldsymbol{\kappa}| x / L) e^{-i t E \boldsymbol{\kappa}},
$$

where $E_{\boldsymbol{\kappa}}=(2 \pi / L)^{2} \sum_{j=1}^{N}\left(\kappa_{j}^{2}+\lambda(N+1-2 j) \kappa_{j}\right)$. The coefficient $\left[0^{\prime}\right]_{\boldsymbol{\kappa}}^{\lambda}$ in Eq. (5.3) vanishes unless the diagram $\mathcal{D}(\boldsymbol{\kappa})$ has no more than $p$ columns of length longer than $q$ and $q$ rows of length longer than p. In other words, the intermediate states contributing to the DDDCF has precisely $p$ hole and $q$ particle excitations. This is a conclusive evidence of the ideal fractional exclusion statistics the CSM quasiparticles and quasiholes obey.

The DDDCF in the thermodynamic limit greatly simplifies as is shown in Appendix A; and it is given by 6 ]

$$
\langle 0|\rho(x, t) \rho(0,0)| 0\rangle=C \prod_{i=1}^{q}\left(\int_{0}^{\infty} d x_{i}\right) \prod_{j=1}^{p}\left(\int_{0}^{1} d y_{j}\right) Q^{2} F\left(q, p, \lambda \mid\left\{x_{i}, y_{j}\right\}\right) \cos (Q x) e^{-i E t}
$$

where $Q$ and $E$, the total momentum and energy, are given in units of $\hbar$ and $\hbar^{2} / 2 m$ by

$$
Q=2 \pi \rho_{0}\left(\sum_{j=1}^{q} x_{j}+\sum_{j=1}^{p} y_{j}\right)
$$




$$
E=\left(2 \pi \rho_{0}\right)^{2}\left(\sum_{j=1}^{q} \epsilon_{P}\left(x_{j}\right)+\sum_{j=1}^{p} \epsilon_{H}\left(y_{j}\right)\right)
$$

with $\rho_{0}=N / L, \epsilon_{P}(x)=x(x+\lambda)$ and $\epsilon_{H}(y)=\lambda y(1-y) . x_{j}\left(\epsilon_{P}\right)$ and $y_{j}\left(\epsilon_{H}\right)$ are normalized momentum (energy) of the quasiparticles and the quasiholes, respectively. The normalization constant $C$ is given by

$$
\begin{aligned}
A(m, n, \lambda) & =\frac{\Gamma^{m}(\lambda) \Gamma^{n}(1 / \lambda)}{\prod_{i=1}^{m} \Gamma^{2}(p-\lambda(i-1)) \prod_{j=1}^{n} \Gamma^{2}(q-(j-1) / \lambda)} \prod_{j=1}^{n}\left(\frac{\Gamma(q-(j-1) / \lambda)}{\Gamma(1-(j-1) / \lambda)}\right)^{2}, \\
C & =\frac{\lambda^{2 p(q-1)} \Gamma^{2}(p)}{2 \pi^{2} p ! q !} A(q, p, \lambda) .
\end{aligned}
$$

Finally, the form factor $F\left(q, p, \lambda \mid\left\{x_{i}, y_{j}\right\}\right)$ is given by

$$
F\left(m, n, \lambda \mid\left\{x_{i}, y_{j}\right\}\right)=\prod_{i=1}^{m} \prod_{j=1}^{n}\left(x_{i}+\lambda y_{j}\right)^{-2} \frac{\left(\prod_{i<j}\left(x_{i}-x_{j}\right)^{2}\right)^{\lambda}\left(\prod_{i<j}\left(y_{i}-y_{j}\right)^{2}\right)^{1 / \lambda}}{\prod_{i=1}^{m} \epsilon_{P}\left(x_{i}\right)^{1-\lambda} \prod_{j=1}^{n} \epsilon_{H}\left(y_{j}\right)^{1-1 / \lambda}} .
$$

The form factor has been conjectured by Haldane based on the clues given by the works of Simons et. al., Galilean invariance, and $U(1)$ conformal field theory [40]. A less general form of the DDDCF at integer values of $\lambda$ has also been reported 41.

The region of support in the energy-momentum space for the DDDCF at $\lambda=5 / 3$ corresponds to the shaded area in Fig. 2. It is obtained by convoluting the dispersion relations of the $q$ quasiparticles and $p$ quasiholes as given by Eqs. (5.5) and (5.6). At lowenergies $2 p$ distinct sectors indicated by darker shade emerge as expected of $1 \mathrm{D}$ metallic system. (A generic 1D system, however, will have an infinite number of these low-energy sectors.)

It is also worth noting that there is a qualitative difference between the finite and the infinite system. The intermediate states represented by the diagrams with $\left|\kappa_{j}-\kappa_{k}\right|$ and $\mid \kappa_{j}^{\prime}-$ $\kappa_{k}^{\prime} \mid$ of order $\mathcal{O}(N)$ are the only states that contribute to the DDDCF in the thermodynamic limit (i.e., $N, L \rightarrow \infty$ with $N / L$ fixed). I call this a super-selection rule [6]. (There can be violations of this rule at the low-energy limit as discussed in Appendix A.) Perhaps, the phase transition that occurs in 2D QCD as $N \rightarrow \infty$ [42] is related to the CSM super-selection rule. 


\section{ONE-PARTICLE GREEN'S FUNCTION}

Unlike the DDDCF calculated in the previous section, evaluation of the one particle Green's function depends on the actual statistics of the real particles since it involves the reordering of the particles. For example, the one-particle density matrix (the static limit of the Green's function) for the $\lambda=1$ case is trivially simple if the ground state $\Psi_{0}$ is taken to be the fermionic wave function. On the other hand, if the wavefunction is taken to describe a bosonic system, the calculation gets quite complicated and different from its fermionic counterpart as shown in 43 .

As described in section $\amalg$ I take the modified CSM given by Eq. (4.7) as one-dimensional anyon system with the exchange phase given by $\exp (i \lambda \pi)$ and Eq. (4.8) as the corresponding wavefunctions. If the $m$ th particle is removed from the ground state of $N+1$ particles, the remaining term after factoring out the ground state wavefunction of the $N$ particle system is

$$
\left(\prod_{j(<m)}\left(z_{j}-z_{m}\right)^{\lambda}\right)\left(\prod_{j(>m)}\left(z_{m}-z_{j}\right)^{\lambda}\right)\left(\prod_{k \neq m} z_{k}^{-\lambda / 2}\right) z_{m}^{-\lambda N / 2} e^{i(m-1) \pi \lambda},
$$

where the last term is determined from the property of the ordering function $\varphi$ given in Eq. (4.9). Therefore, up to an overall phase factor that does not depend on the position of the removed particle, the destruction operation $\Psi(x)$ on the ground state of $N+1$ particles is given by

$$
\Psi(x)|0\rangle_{N+1}=\frac{A_{N+1}}{A_{N}} z^{-\lambda N / 2} \prod_{j=1}^{N}\left(z-z_{j}\right)^{\lambda} z_{j}^{-\lambda / 2}|0\rangle_{N},
$$

where $z=\exp (i 2 \pi x / L)$ and $A_{N}^{2}=(1 / L)^{N} \Gamma^{N}(1+\lambda) / \Gamma(1+\lambda N)$. The statistical phase

arising from the ordering function makes the destruction operation symmetric with respect to the permutations of $\left\{z_{j}\right\}$. The symmetric function $\prod_{j=1}^{N}\left(z-z_{j}\right)^{\lambda}$ can then be expanded in term of Jack polynomials using Eqs. (3.10) and (3.11).

The hole propagator part of the one-particle Green's function is defined as

$$
\begin{aligned}
{ }_{N+1}\left\langle 0\left|\Psi^{\dagger}\left(x^{\prime}, t^{\prime}\right) \Psi(x, t)\right| 0\right\rangle_{N+1} & ={ }_{N+1}\left\langle 0\left|e^{i H_{N+1} t^{\prime}} \Psi^{\dagger}\left(x^{\prime}\right) e^{-i H_{N} t^{\prime}} e^{i H_{N} t} \Psi(x) e^{-i H_{N+1} t}\right| 0\right\rangle_{N+1} \\
& ={ }_{N+1}\left\langle 0\left|\Psi^{\dagger}\left(x^{\prime}\right) e^{-i\left(\left(H_{N}-E_{N}\right)-\mu\right)\left(t^{\prime}-t\right)} \Psi(x)\right| 0\right\rangle_{N+1}
\end{aligned}
$$


where the chemical potential $\mu=E_{N+1}-E_{N}$. By expanding $\Psi(x)$ in terms of Jack polynomials and using the orthogonality relation (3.7) the propagator at finite $N$ and $L$ is evaluated to be

$$
\begin{aligned}
{ }_{N+1}\left\langle 0\left|\Psi^{\dagger}(x, t) \Psi(0,0)\right| 0\right\rangle_{N+1} & =(N+1)\left(\frac{A_{N+1}}{A_{N}}\right)^{2} \\
& \times \sum_{\boldsymbol{\kappa}} \frac{\lambda^{-2|\boldsymbol{\kappa}|}}{j_{\boldsymbol{\kappa}}^{\lambda}} \frac{\left(\{-\lambda\}_{\boldsymbol{\kappa}}^{\lambda}\right)^{2}[N]_{\boldsymbol{\kappa}}^{\lambda}}{[N+1 / \lambda-1]_{\boldsymbol{\kappa}}^{\lambda}} e^{i 2 \pi(|\boldsymbol{\kappa}|-\lambda N / 2) x / L} e^{-i\left(E \boldsymbol{\kappa}^{-\mu) t}\right.},
\end{aligned}
$$

where the additional $(N+1)$ factor comes from the freedom of choosing one of $N+1$ available particles to destroy and create. The coefficient $\{-\lambda\}_{\boldsymbol{\kappa}}^{\lambda}$ vanishes unless the diagram $\mathcal{D}(\boldsymbol{\kappa})$ has at most $q-1$ rows of length greater than $p$ and $p$ columns of length greater than $q-1$. Therefore, the intermediate states for the propagator is spanned by $q-1$ quasiparticles and $p$ quasiholes. This is a very important result. The exclusion statistics of the quasiparticles and quasiholes is completely consistent with the anyon statistics of the real particles. [6]

Taking the thermodynamic limit of Eq. (6.4) is almost identical to that of Eq. (5.3) and is given by [6]

$$
\left\langle 0\left|\Psi^{\dagger}(x, t) \Psi(0,0)\right| 0\right\rangle=\rho_{0} D \prod_{i=1}^{q-1}\left(\int_{0}^{\infty} d x_{i}\right) \prod_{j=1}^{p}\left(\int_{0}^{1} d y_{j}\right) F\left(q-1, p, \lambda \mid\left\{x_{i}, y_{j}\right\}\right) e^{i\left(\left(Q-Q_{0}\right) x-(E-\mu) t\right)}
$$

where the chemical potential $\mu=\left(\pi \lambda \rho_{0}\right)^{2}$ and the back flow $Q_{0}=\pi \lambda \rho_{0} . F\left(q-1, p, \lambda \mid\left\{x_{i}, y_{j}\right\}\right)$ is still given by Eq. (5.9) and D by

$$
D=\frac{\lambda^{2 p(q-1)} \Gamma^{2}(p)}{\Gamma(\lambda)(q-1) ! p !} A(q-1, p, \lambda)
$$

$Q$ and $E$ are same as before except for the number of $x_{j}$ 's. At integer values of $\lambda$ (i.e., $q=1$ case where only quasiholes are excited), based on the equal-time results of Forrester 18 Haldane made a conjecture [5] which agrees with this formula. The regions of support for the hole propagator is given by the shaded area in $Q>0$ (or $Q<0$ ) in Fig. 2. There are also shifts in $E$ and $Q$ by $-\mu$ and $-Q_{0}$, respectively.

It is also interesting to consider the following function 


$$
\Psi_{m}^{\lambda}(x)=\prod_{i<j}\left(z-z_{j}\right)^{m},
$$

where $m$ is a positive integer. This function can be expanded in terms of Jack polynomials using Eq. (3.10) with coefficients containing the term $\{-m\}_{\boldsymbol{\kappa}}^{\lambda}$ which vanishes unless the diagram $\mathcal{D}(\boldsymbol{\kappa})$ has no more than $m$ columns and no rows longer than $m$. Therefore, $\Psi_{m}^{\lambda}(x)$

acting on the ground state creates exactly $m$ quasiholes. $\Psi_{m}^{\lambda}(x)$ is a generalization of the one-hole state given in Eq. (2.9). The propagator can easily be calculated and its form factor is given by $F\left(0, m, \lambda \mid\left\{y_{j}\right\}\right)$. This result is consistent with the conjecture [6] that the minimal form factor for any two point correlation functions whose intermediate states contain only $n$ quasiparticle and $m$ quasiholes is given by $F\left(n, m, \lambda \mid\left\{x_{i}, y_{j}\right\}\right)$.

\section{LOW-ENERGY EFFECTIVE THEORY AND COUPLED FRACTIONAL QUANTUM HALL EDGE STATES}

Following the standard method in Luttinger liquid theory [44] I construct an effective low-energy model for the 1D anyon system. When $\lambda$ is an integer this system is equivalent to a coupled system of left- and right-moving edge states of the FQHE. The excitations on a single edge of the FQH fluid moves only in one direction because of the externally applied magnetic field; and they have been rather thoroughly studied using the so called chiral Luttinger liquid theory [24] which is intrinsically anomalous [45]. When there is an extra edge on the FQH droplet (e.g. a strip, annulus, or cylindrical geometry instead of the disk geometry) and when the edges are close enough, new phase space opens up as a result of the fractional charge transfer between the edges. There actually have been many suggestions that the CSM is related to the edge states [21]. In this section I show in another way that the CSM is an exactly solvable 1D anyon system by calculating the correlation functions of the effective model and finding exact agreement with those of the CSM in the long-wavelength limit.

A general gauge invariance argument [46] can be used to map out the qualitative structure of the excitation spectra in the energy-momentum space. I use the cylindrical geometry [46] 
with coordinates $x$ and $y$ defined as shown in Fig. 3. I put some incompressible fluid perhaps made up of $\lambda$ anyons on the surface of the cylinder with a confining potential in $y$-direction such that two edges are created along $x$-direction. (Of course, when $\lambda$ is not an integer the edges are not related to the FQHE edges which are more complicated composite type [24].) The cylinder with circumference $L$ is also pierced by a thin solenoid along its longitudinal axis, thus inducing a magnetic flux $\Phi=A L$ through its cross-section and a vector potential $\vec{A}=A \hat{x}$ on its surface. There is also magnetic fields $\vec{B}$ normal to the entire surface of the cylinder. Since the left- and right-moving sectors of the CSM completely decouple in the low-energy limit, this geometrical construction is in fact equivalent to the CSM and is useful to show intuitively how the low-energy sectors are related.

The fictitious flux applied to the cylinder is used as a passive device for mapping out the regions of low-energy excitation sectors of the coupled anyon edges. By virtue of the gauge invariance the energy is degenerate at flux equal to $2 \pi n$ (in units where $\hbar=e=1$ ) which corresponds to the momentum of $2 \pi n \rho_{0}$. The adiabatical change in flux from 0 to $2 \pi$ induces an elementary excitation carrying charge $-q / p$ and flux $-2 \pi$ (i.e., a quasihole) to move from one edge to the other; and, in between, it costs finite energy to the system since the bulk is incompressible. In fact the real space configuration of an incompressible droplet is equivalent to a "Fermi sea." [47] The edges of the fluid coincide with the locus of points in which Fermi energy crosses the external confining potential. The only degrees of freedom left in "Fermi sea" in the low-energy limit is the "Fermi surface" fluctuation. This "Fermi sea" obeys the fractional exclusion statistics. Since there is a symmetry along $x$-direction, the fluid can be regarded as an 1D "Fermi sea" and the left- and the right-edges as two "Fermi points."

In light of the observations made so far it is reasonable to model the coupled edges by a full (non-chiral) 1D anyon system. First, I construct the one-dimensional anyon creation operator as follow

$$
\Psi_{\lambda}^{\dagger}(x)=\Psi_{B}^{\dagger}(x) e^{i \lambda \theta(x)}
$$


where $\Psi_{B}^{\dagger}$ is the boson creation operator and $\lambda$ the statistical parameter. The operator $e^{i \lambda \theta(x)}$ is the so called "disorder operator" 48 that creates a kink (or vortex) of size $\pi \lambda$ at position $x$ and is defined as

$$
\theta(x)=\pi \int_{-\infty}^{x} \rho\left(x^{\prime}\right) d x^{\prime}
$$

where $\rho(x)$ is the density operator. Therefore, $\Psi_{\lambda}^{\dagger}(x)$ is a composite operator that creates a boson plus a vortex; so, it is an anyon creation operator.

The boson creation operator $\Psi_{B}^{\dagger}(x)$ for a system with multiple low-energy sectors has previously been constructed 49 and is given by

$$
\Psi_{B}^{\dagger}(x) \approx \rho_{0}^{1 / 2} \sum_{m=-\infty}^{+\infty} e^{i 2 m \theta(x)} e^{i \phi(x)}
$$

where the phase field $\phi(x)$ is defined by the following canonical commutation relation: $\left[\phi(x), \rho\left(x^{\prime}\right)\right]=i \delta\left(x-x^{\prime}\right)$. The multi-sector density operator is also given by

$$
\tilde{\rho}(x)=\rho(x) \sum_{m=-\infty}^{+\infty} e^{i 2 m \theta(x)} .
$$

Here, the sectors are connected by the operator $\exp (i 2 m \theta(x))$ which creates a vortex of size $2 \pi m$. This dynamical device now replaces the passive device I previously used to map the system from one sector to the next by supplying external magnetic flux to the cylinder.

The fields $\theta(x), \phi(x)$ and $\rho(x)$ can now be expressed in terms of Tomonaga boson operators $b^{\dagger}$ and $b$ as [49,50]

$$
\begin{aligned}
& \theta(x)=\theta_{0}+\pi \rho_{0} x+i e^{\varphi} \sum_{k \neq 0} \frac{1}{k}\left|\frac{\pi k}{2 L}\right|^{1 / 2} e^{-i k x}\left(b_{-k}^{\dagger}+b_{k}\right) \\
& \phi(x)=\phi_{0}-i e^{-\varphi} \sum_{k \neq 0}\left|\frac{\pi}{2 k L}\right|^{1 / 2} e^{-i k x}\left(b_{-k}^{\dagger}-b_{k}\right) \\
& \rho(x)=\rho_{0}+e^{\varphi} \sum_{k \neq 0}\left|\frac{k}{2 \pi L}\right|^{1 / 2} e^{-i k x}\left(b_{-k}^{\dagger}+b_{k}\right) .
\end{aligned}
$$

I assume here that the most important interaction is the statistical interaction; so, I can set the Bogoliubov parameter $e^{2 \varphi}=1 / \lambda$.

Now, $\Psi_{\lambda}^{\dagger}(x)$ can easily be shown to satisfy the following anyon commutation relation 


$$
\Psi_{\lambda}^{\dagger}(x) \Psi_{\lambda}^{\dagger}\left(x^{\prime}\right)=e^{i \pi \lambda \operatorname{sgn}\left(x^{\prime}-x\right)} \Psi_{\lambda}^{\dagger}\left(x^{\prime}\right) \Psi_{\lambda}^{\dagger}(x) \quad \text { for } x \neq x^{\prime}
$$

Eq. (7.8) can thus be used as a defining relation for the one-dimensional anyons.

The Hamiltonian is diagonal in Tomonaga boson operators and is given as usual by

$$
H=\hbar v_{s} \sum_{q \neq 0}|q| b_{q}^{\dagger} b_{q}
$$

where $v_{s}$ is the sound velocity.

Using the operator identities (i) $e^{A} e^{B}=\exp \left(e^{\alpha}-1\right) e^{B} e^{A}$ if $[A, B]=\alpha B$, (ii) $e^{A} e^{B}=$ $\exp (-[A, B]) e^{B} e^{A}$ and $e^{A+B}=\exp (-[A, B] / 2) e^{A} e^{B}$ if $[[A, B], A]=[[A, B], B]=0$, the following correlation functions are calculated

$$
\begin{aligned}
\langle\hat{\rho}(x, t) \hat{\rho}(0,0)\rangle & \approx \rho_{0}^{2}\left(1-\frac{\lambda^{-1}}{\left(2 \pi \rho_{0}\right)^{2}}\left(\frac{1}{\left(\xi^{-}\right)^{2}}+\frac{1}{\left(\xi^{+}\right)^{2}}\right)\right. \\
& \left.+\sum_{m=1}^{\infty} A_{m}\left(\frac{1}{\xi^{+} \xi^{-}}\right)^{m^{2} / \lambda} \cos \left(2 \pi \rho_{0} m x\right)\right) \\
\left\langle\Psi_{\lambda}^{\dagger}(x, t) \Psi_{\lambda}(0,0)\right\rangle & \approx \rho_{0} \sum_{m=-\infty}^{\infty} B_{m}\left(\frac{1}{\xi^{+}}\right)^{(m+\lambda)^{2} / \lambda}\left(\frac{1}{\xi^{-}}\right)^{m^{2} / \lambda} e^{i\left(2 \pi \rho_{0}(m+\lambda / 2) x+\mu t\right)}
\end{aligned}
$$

where $\xi^{ \pm}=x \mp v_{s} t, \mu$ the chemical potential and the coefficients $A_{m}\left(B_{m}\right)$ are regularizationdependent constants.

The Green's function $\left\langle\Psi_{\lambda}^{\dagger}(x, t) \Psi_{\lambda}(0,0)\right\rangle$ for the sector $m=0$, where the charge transfer from one edge to the other is forbidden, is given by only the right-movers (or only the left-movers if $\Psi_{\lambda}^{\dagger}$ were properly redefined) even though the anyon creation operator $\Psi_{\lambda}^{\dagger}(x)$ contains both the right- and the left-moving bosonic modes (i.e., $b_{k}^{\dagger}$ and $b_{k}$ for both $k>0$ and $k<0$ ). This chiral sector emerges naturally in this theory without explicitly imposing the chirality condition.

In an isolated chiral theory coupled to a gauge field the charge is in general not conserved and because of this the theory is known to be anomalous and not physical. As far as the isolated chiral theory is concerned the only physical sector is the chiral sector where no charge transfer between the edges is possible. One can, however, consider an "almost" chiral theory which describes a system of two chiral edge states that are independent except 
for the charge leakage from one side to the other and vice versa. For the sake of definiteness I concentrate on the right edge and propose the "almost" chiral system with the following Hamiltonian and the field operators,

$$
\begin{aligned}
H^{R} & =\hbar v_{s} \sum_{q>0} q b_{q}^{\dagger} b_{q}, \\
\theta^{R}(x) & =\theta_{0}+\pi \rho_{0}^{R} x-\frac{i}{\sqrt{\lambda}} \sum_{k>0} \frac{1}{k}\left|\frac{\pi k}{2 L}\right|^{1 / 2}\left(e^{i k x} b_{k}^{\dagger}-e^{-i k x} b_{k}\right), \\
\phi^{R}(x) & =\phi_{0}-i \sqrt{\lambda} \sum_{k>0}\left|\frac{\pi}{2 k L}\right|^{1 / 2}\left(e^{i k x} b_{k}^{\dagger}-e^{-i k x} b_{k}\right), \\
\Psi_{\lambda}^{R^{\dagger}}(x) & \approx \sqrt{\rho_{0}^{R}} \sum_{m} e^{i 2(m+\lambda / 2) \theta^{R}(x)} e^{i \phi^{R}(x)} .
\end{aligned}
$$

The chiral system is constructed only with the right-moving Tomonaga bosons. The Green's function in this case is given by

$$
\left\langle\Psi_{\lambda}^{R^{\dagger}}(x, t) \Psi_{\lambda}^{R}(0,0) \approx \rho_{0}^{R} \sum_{m=-\infty}^{\infty} C_{m}\left(\frac{1}{x-v_{s} t}\right)^{(m+\lambda) / \lambda} e^{i\left(2 \pi \rho_{0}^{R}(m+\lambda / 2) x+\mu^{R} t\right)} .\right.
$$

The $m=0$ sector here is equivalent to the corresponding chiral sector of the non-chiral model. As expected only the right-movers contribute to the Green's function for all the other sectors.

Asymptotic expansions of the correlation functions of the CSM have been calculated in Appendix B; and they agree with Eq. (7.10) and Eq. (7.11). This agreement between the correlation functions of the explicitly constructed anyon model and the CSM shows that the first- and the second-quantized construction of the anyons are in fact equivalent in the long-wavelength limit.

\section{FINITE-SIZE SCALING AND CORRELATION EXPONENTS}

There is another extremely elegant and powerful way of obtaining the exponents of the correlation functions in the long-wavelength limit. If the dispersion relations of the elementary excitations of the one-dimensional quantum model in the low-energy limit is linear (i.e., has Lorentz as well as Galilean invariance so that the space and time variables 
are on equal footing), the principle of conformal invariance is applicable and, in particular, the finite-size corrections to the energy and momentum become universal and are directly related to the exponents of the correlation functions [51].

The finite-size scaling has previously been applied to the CSM by Kawakami and Yang [52] without the benefit of recently uncovered knowledge [5].6], namely that due to the ideal fractional statistics the CSM particles obey the intermediate states for the DDDCF and the Green's function are spanned by finite number of the elementary excitations. The shaded regions in Fig. 22 are the relevant sectors for the correlation functions (for the Green's function only the positive or negative momentum sectors contribute). A complete set of relevant exponents is obtained using the finite-size scaling in this section.

Let $\left(p_{L}, h_{L} \mid p_{R}, h_{R}\right)$ be a label for a low-energy sector spanned by $p_{L}\left(h_{L}\right)$ left-moving and $p_{R}\left(h_{R}\right)$ right-moving quasiparticles (quasiholes). The relevant sectors for the DDDCF are $(0, n \mid q, p-n)$ and $(q, p-n \mid 0, n)$ and for the Green's function $(0, n \mid q, p-n)$ or $(q, p-n \mid 0, n)$ where $n=0,1, \ldots, p$. First, for the DDDCF the lowest-energy state in the sector $m$ is characterized by $m$ quasiholes on right (or left) side of the "Fermi sea" transfered to the left (or right). Hence, the pseudomomenta $k_{j}$ for this sector is given in terms of the ground-state pseudomomenta $k_{j}^{0}$ as $k_{j}=k_{j}^{0}+2 \pi m / L$; and the energy and momentum by

$$
\begin{aligned}
& E_{m}=E_{0}+\frac{2 \pi v_{s}}{L} \frac{m^{2}}{\lambda}, \\
& P_{m}=2 \pi m \rho_{0},
\end{aligned}
$$

where the sound velocity $v_{s}=2 \pi \lambda \rho_{0}$. The scaling dimension $x=h^{+}+h^{-}$of the density operator and its conformal spin $s=h^{+}-h^{-}$are therefore given by $x=m^{2} / \lambda$ and $s=0$, where $h^{ \pm}$are right (left) conformal weights and $2 h^{ \pm}$are the exponents that actually appear in the correlation functions for the right(left) movers. Thus, $2 h^{+}=2 h^{-}=m^{2} / \lambda$ in agreement with the exponents found in the previous section and in Appendix B.

Now, for the hole propagator the energy and momentum of the sector $m$ characterized by $m$ quasihole transfers from the left to right "Fermi points" and thus the pseudomomenta $k_{j}=k_{j}^{0}+2 \pi m / L$, where $j=1, \ldots, N-1$, are 


$$
\begin{aligned}
& E_{m}=E_{0}-\mu+\frac{2 \pi v_{s}}{L}\left(\frac{m^{2}}{\lambda}-n+\frac{\lambda}{2}\right), \\
& P_{m}=2 \pi(n-\lambda / 2) \rho_{0}+\frac{2 \pi}{L}\left(\frac{\lambda}{2}-n\right) .
\end{aligned}
$$

The chemical potential $\mu=\left(\pi \lambda \rho_{0}\right)^{2}$ is associated with the particle destruction. I remove the

rightmost pseudoparticle with $k_{N}^{0}=\pi \lambda(N-1) / L$ from the ground state condensate and do not introduce any separate selection rules in contrast to Ref. [52]. In this case the right and left conformal weights are different (due to the non-zero conformal spin $s=\lambda / 2-n$ ) and are given by $2 h^{+}=(m-\lambda)^{2} / \lambda$ and $2 h^{-}=m^{2} / \lambda$ as expected. The right and left conformal weights would be switched if the excitations were caused by the removal of the leftmost pseudoparticle at $-\pi \lambda(N-1) / L$.

Apparently, the long-range interaction does not destroy the conformal invariance of the CSM in the long-wavelength limit. This is expected from the linear dispersion relations of the CSM quasiparticles and quasiholes in this limit.

\section{LATTICE MODELS}

The Haldane-Shastry model (HSM) corresponds to a lattice generalization of the CSM at $\lambda=2$ and has the following Hamiltonian

$$
H=J_{0} \sum_{i<j} \frac{\vec{S}_{i} \cdot \vec{S}_{j}}{d^{2}(i-j)},
$$

where $d(m)=(N / \pi)|\sin (\pi m / N)|$ and $\vec{S}_{j}$ is the $S U(2)$ spin operator acting on site $j$. The model possesses a quantum symmetry called Yangian [8] and exhibits supermultiplets structures whose spin contents are exactly reproducible from an asymptotic limit of the thermodynamic Bethe-ansatz equations [12]. Furthermore, there is one-to-one correspondence between the highest weight states of the Yangian supermultiplets and the states of the CSM (i.e., they satisfy the same eigenvalue equations.) A further generalization to $S U(n)$ case has also been accomplished in [10,11]. The lattice CSM model at even (odd) integer values of $\lambda$ can be mapped to bosonic (fermionic) spinless t-J model [10]. 
The method for evaluating the correlation functions of the CSM presented in this paper is directly applicable to its lattice cousins in some limited cases. The Galilean invariance is broken in the lattice models and is replaced with much weaker lattice translation symmetry which induces appearance of the Brillouin zone boundaries. If the elementary excitations created by a local operator acting on the ground state do not cross the Brillouin zone boundaries and the excited states are the Yangian highest weight states, then the corresponding correlation functions of HSM are identical to that of the CSM.

\section{CONCLUSION}

In this paper the fractional exchange and exclusion statistics are studied using the exactly solvable Calogero-Sutherland model; and they are found to be mutually consistent. I have shown that the interaction giving rise to the fractional exclusion statistics for the elementary excitations of a given condensed matter system can in fact be treated as the statistical gauge field carried by the real particles making up the system. This was done by calculating the exact dynamical density-density correlation function and one-particle Green's function using Jack symmetric polynomials and examining the intermediate states contributing to the correlation functions. I find that the intermediate states for $\lambda=p / q$ CSM are spanned by $q$ quasiparticles and $p$ quasiholes for the density-density correlation function and $q-1$ quasiparticles and $p$ quasiholes for the hole propagator and thereby show that the quasiparticles indeed carry charge 1 and flux $2 \pi \lambda$ and the quasiholes charge $-1 / \lambda$ and flux $-2 \pi$ as first suggested by Haldane [5].

I also construct an explicit multi-sector anyon operators in analogy with Haldane's harmonic-fluid 499 and calculate their correlation functions which agree with those of the CSM which corresponds to the first-quantized construction of anyons. Therefore, the CSM at odd-integer coupling constant describes the edge states of the fractional quantum Hall droplet corresponding to the Laughlin states as suggested in Ref. [21].

There are some interesting open problems: 
- How can one rigorously construct 1D fractional exchange statistics using the YangBaxter equation?

- For the CSM with the coupling constant other than $\lambda=1 / 2,1$, and 2 , are there any corresponding random matrices?

- How can one generalize Jack polynomials to the $S U(n)$ case?

I hope to see some of these questions answered in the future.

\section{ACKNOWLEDGMENT}

This work is supported by DOE grant DE-FG02-90ER40542.

\section{APPENDIX A: HOW TO TAKE THE THERMODYNAMIC LIMIT}

The thermodynamic limit of the DDDCF given by Eq. (5.3) greatly simplifies by the fact that the coefficient $\left[0^{\prime}\right]_{\boldsymbol{\kappa}}^{\lambda}$ vanishes unless the diagram $\mathcal{D}(\boldsymbol{\kappa})$ has at most $p$ rows of length longer than $q$ and $q$ columns of length longer than $p$. As shown in Section $\mathbb{\nabla}$ the minimal charge neutral excitations in the CSM consist of $q$ quasiparticles and $p$ quasiholes; therefore, the intermediate states that contribute to the correlation function have exactly $q$ quasiparticle and $p$ quasihole excitations.]

The contributing diagrams $\mathcal{D}(\boldsymbol{\kappa})$, for convenience, are divided into three subdiagrams $\mathcal{A}(\boldsymbol{\kappa})=\{(i, j), 1 \leq i \leq p, 1 \leq j \leq q\}, \mathcal{B}(\boldsymbol{\kappa})=\left\{(i, j), 1 \leq j \leq q, q+1 \leq i \leq \kappa_{j}^{\prime}\right\}$ and $\mathcal{C}(\boldsymbol{\kappa})=\left\{(i, j), 1 \leq i \leq p, p+1 \leq j \leq \kappa_{i}\right\}$ as illustrated in Fig. 6 . The factors expressed in terms of the generalized factorial over $\mathcal{D}(\boldsymbol{\kappa})$ are evaluated for each of the subdiagrams, separately. Then, later the each subfactors are multiplied to obtain to full factors over the diagram $\mathcal{D}$.

\footnotetext{
${ }^{1}$ The non-vanishing diagrams with less than $p$ rows and $q$ columns are interpreted as having some of the quasiparticles and quasiholes in their unexcited modes.
} 
First, consider the following factor that appears in the DDDCF

$$
F_{1}=\prod_{(i, j) \in \boldsymbol{\kappa}}\left(\frac{N+(j-1) / \lambda-(i-1)}{N+j / \lambda-i}\right) .
$$

$F_{1}$ over $\mathcal{A}$ simplifies in the thermodynamic limit to

$$
F_{1}(\mathcal{A})=\prod_{j=1}^{p} \prod_{i=1}^{q}\left(\frac{N+(j-1) / \lambda-(i-1)}{N+j / \lambda-i}\right) \stackrel{N \rightarrow \infty}{\rightarrow} 1 .
$$

In order to evaluate $F_{1}$ over $\mathcal{B}$ I rewrite the product in terms of the gamma functions using the identity $\Gamma(z+n) / \Gamma(z)=z(z+1) \cdots(z+n-1)$ as

$$
\begin{aligned}
F_{1}(\mathcal{B}) & =\prod_{j=1}^{p} \frac{\left(N+(j-1) / \lambda-\kappa_{j}^{\prime}+1\right) \cdots(N+(j-1) / \lambda-q)}{\left(N+j / \lambda-\kappa_{j}^{\prime}\right) \cdots(N+j / \lambda-q-1)} \\
& =\prod_{j=1}^{p} \frac{\Gamma\left(N+j / \lambda-\kappa_{j}^{\prime}\right) \Gamma(N+j / \lambda-q+1-1 / \lambda)}{\Gamma\left(N+j / \lambda-\kappa_{j}^{\prime}+1-1 / \lambda\right) \Gamma(N+j / \lambda-q)} .
\end{aligned}
$$

Using the following asymptotic relation,

$$
\lim _{|z| \rightarrow \infty} \frac{\Gamma(z+a)}{\Gamma(z)}=z^{a}
$$

I reduce $F_{1}(\mathcal{B})$ in Eq. $(\mathrm{A} 3)$ as

$$
\begin{aligned}
F_{1}(\mathcal{B}) & \stackrel{N \rightarrow \infty}{\rightarrow} \prod_{j=1}^{p}\left(\frac{N+j / \lambda-q}{N+j / \lambda-\kappa_{j}^{\prime}}\right)^{1-1 / \lambda} \\
& \rightarrow \prod_{j=1}^{p}\left(1-\kappa_{j}^{\prime} / N\right)^{1 / \lambda-1}
\end{aligned}
$$

$F_{1}$ over $\mathcal{C}$, similarly, is given by

$$
F_{1}(\mathcal{C}) \stackrel{N \rightarrow \infty}{\rightarrow} \prod_{i=1}^{q}\left(1+\frac{1}{\lambda} \frac{\kappa_{i}}{N}\right)^{\lambda-1}
$$

Evaluation of $\left(\left[0^{\prime}\right]_{\boldsymbol{\kappa}}^{\lambda}\right)^{2}$ is straightforward and is equal to product of the following three factors

$$
\begin{aligned}
\left(\left[0^{\prime}\right]_{\boldsymbol{\kappa}}^{\lambda}\right)^{2}(\mathcal{A}) & =\lambda^{-2(p-1)} \Gamma^{2}(p) \prod_{j=1}^{p} \frac{\Gamma^{2}(q-(j-1) / \lambda)}{\Gamma^{2}(1-(j-1) / \lambda)}, \\
\left(\left[0^{\prime}\right]_{\boldsymbol{\kappa}}^{\lambda}\right)^{2}(\mathcal{B}) & =\prod_{j=1}^{p} \frac{\Gamma^{2}\left(-(j-1) / \lambda+\kappa_{j}^{\prime}\right)}{\Gamma^{2}(-(j-1) / \lambda+q)}, \\
\left(\left[0^{\prime}\right]_{\boldsymbol{\kappa}}^{\lambda}\right)^{2}(\mathcal{C}) & =\lambda^{-2\left(\sum_{i=1}^{q} \kappa_{i}\right)+2 p q} \prod_{i=1}^{q} \frac{\Gamma^{2}\left(\kappa_{i}-\lambda(i-1)\right)}{\Gamma^{2}(p-\lambda(i-1))} .
\end{aligned}
$$


The value of $j_{\boldsymbol{\kappa}}^{\lambda}$ over $\mathcal{A}$ is given by

$$
j_{\boldsymbol{\kappa}}^{\lambda}(\mathcal{A})=\prod_{i=1}^{q} \prod_{j=1}^{p}\left(\kappa_{j}^{\prime}+\frac{1}{\lambda} \kappa_{i}\right)^{2} .
$$

In order to evaluate $j_{\boldsymbol{\kappa}}^{\lambda}$ over $\mathcal{B}$, I further divide up the subdiagram $\mathcal{B}$ into $p$ cells such that $l$ th cell is given by $\left\{(i, j), 1 \leq j \leq l, \kappa_{l+1}^{\prime}+1 \leq i \leq \kappa_{l}^{\prime}\right\}$. If $\kappa_{l}^{\prime}=\kappa_{l+1}^{\prime}$, then the $l$ th cell is empty. Fig. 5 illustrates how the subdiagram $\mathcal{B}$ is divided into $p$ cells and how the empty cells appear. The value of $j_{\boldsymbol{\kappa}}^{\lambda}$ over $\mathcal{B}$ is then as follow

$$
\begin{aligned}
j_{\boldsymbol{\kappa}}^{\lambda}(\mathcal{B}) & =\prod_{l=1}^{p} \prod_{j=1}^{l} \prod_{i=\kappa_{l+1}^{\prime}+1}^{\kappa_{l}^{\prime}}\left(\kappa_{j}^{\prime}-i+1+(l-j) / \lambda\right)\left(\kappa_{j}^{\prime}-i+(l-j+1) / \lambda\right) \\
& =\prod_{l=1}^{p} \prod_{j=1}^{l} \frac{\Gamma\left(\kappa_{j}^{\prime}-\kappa_{l+1}^{\prime}+1+(l-j) / \lambda\right) \Gamma\left(\kappa_{j}^{\prime}-\kappa_{l+1}^{\prime}+(l-j+1) / \lambda\right)}{\Gamma\left(\kappa_{j}^{\prime}-\kappa_{l}^{\prime}+1+(l-j) / \lambda\right) \Gamma\left(\kappa_{j}^{\prime}-\kappa_{l}^{\prime}+(l-j+1) / \lambda\right)},
\end{aligned}
$$

where $\kappa_{p+1}^{\prime} \equiv q$. The contributions from the empty cells are identically equal to one. For the non-empty $l$ th cell $\kappa_{i}=l$ for $\kappa_{l+1}^{\prime}+1 \leq i \leq \kappa_{l}^{\prime}$. The expression above simplifies further to

$$
\begin{aligned}
j_{\boldsymbol{\kappa}}^{\lambda}(\mathcal{B}) & =\frac{1}{\Gamma^{p}(1 / \lambda)} \prod_{j=1}^{p} \Gamma\left(\kappa_{j}^{\prime}+1-j / \lambda\right) \Gamma\left(\kappa_{j}^{\prime}-(j-1) / \lambda\right) \\
& \times \prod_{i>j} \frac{\Gamma\left(\kappa_{i}^{\prime}-\kappa_{j}^{\prime}+1-(i-j+1) / \lambda\right) \Gamma\left(\kappa_{i}^{\prime}-\kappa_{j}^{\prime}-(i-j) / \lambda\right)}{\Gamma\left(\kappa_{i}^{\prime}-\kappa_{j}^{\prime}+1-(i-j) / \lambda\right) \Gamma\left(\kappa_{i}^{\prime}-\kappa_{j}^{\prime}-(i-j-1) / \lambda\right)} .
\end{aligned}
$$

In order to take the $N \rightarrow \infty$ limit using Eq. (ब4), I take the following ratio first and then send the ratio to the limit,

$$
\frac{\left(\left[0^{\prime}\right]_{\boldsymbol{\kappa}}^{\lambda}\right)^{2}(\mathcal{B})}{j_{\boldsymbol{\kappa}}^{\lambda}(\mathcal{B})} \stackrel{N \rightarrow \infty}{\rightarrow} \frac{\Gamma^{p}(1 / \lambda)}{\prod_{j=1}^{p} \Gamma^{2}(q-(j-1) / \lambda)} \prod_{j=1}^{p}\left(\kappa_{j}^{\prime}\right)^{1 / \lambda-1} \prod_{i>j}\left|\kappa_{i}^{\prime}-\kappa_{j}^{\prime}\right|^{2 / \lambda}
$$

Similarly, $j_{\boldsymbol{\kappa}}^{\lambda}$ over $\mathcal{C}$ is given by

$$
\begin{aligned}
j_{\boldsymbol{\kappa}}^{\lambda}(\mathcal{C}) & =\frac{\lambda^{-2\left(\sum_{i=1}^{q} \kappa_{i}\right)+2 p q}}{\Gamma^{q}(\lambda)} \prod_{i=1}^{q} \Gamma\left(\kappa_{i}-\lambda(i-1)\right) \Gamma\left(\kappa_{i}+1-\lambda i\right) \\
& \times \prod_{j>i} \frac{\Gamma\left(\kappa_{i}-\kappa_{j}-\lambda(i-j)\right) \Gamma\left(\kappa_{i}-\kappa_{j}+1-\lambda(i-j+1)\right)}{\Gamma\left(\kappa_{i}-\kappa_{j}-\lambda(i-j-1)\right) \Gamma\left(\kappa_{i}-\kappa_{j}+1-\lambda(i-j)\right)} .
\end{aligned}
$$

I take the following ratio and sent it to the thermodynamic limit using Eq. (A4),

$$
\frac{\left(\left[0^{\prime}\right]_{\boldsymbol{\kappa}}^{\lambda}\right)^{2}(\mathcal{C})}{j_{\boldsymbol{\kappa}}^{\lambda}(\mathcal{C})} \stackrel{N \rightarrow \infty}{\rightarrow} \frac{\Gamma^{q}(\lambda)}{\prod_{i=1}^{q} \Gamma^{2}(p-\lambda(i-1))} \prod_{j=1}^{p} \kappa_{j}^{\lambda-1} \prod_{i>j}\left|\kappa_{i}-\kappa_{j}\right|^{2 \lambda}
$$


Putting all the terms together, setting $\kappa_{j} / N=x_{j}$ and $\kappa_{j}^{\prime} / N=y_{j}$ and turning the sums into integrals I get Eq. (5.4) with the normalization constant given by Eq. (5.8).

If the differences $\left|\kappa_{i}^{\prime}-\kappa_{j}^{\prime}\right|\left(\left|\kappa_{i}-\kappa_{j}\right|\right)$ are of order $\mathcal{O}(1)$ then the corresponding contributions to Eq. (A13) (Eq. (A15)) are of order $\mathcal{O}(1)$ instead of $\mathcal{O}\left(N^{2 / \lambda}\right)\left(\mathcal{O}\left(N^{2 \lambda}\right)\right)$ as $N \rightarrow \infty$;

therefore, the contributions of such terms to the DDDCF are suppressed in the thermodynamic limit. If $\kappa_{j}^{\prime}$ is of order $\mathcal{O}(1)$ or $\mathcal{O}(N)$ then there is a corresponding contribution of order $\mathcal{O}\left(N^{1-1 / \lambda}\right)$; so, if $\lambda<1$ the form factor vanishes as $N \rightarrow \infty$, and if $\lambda>1$ it diverges. However, the net contribution of such term to the DDDCF is of order $N^{-1} \times N^{1-1 / \lambda}$ which always vanishes so long as $\lambda \geq 0^{+}$. (The factor $1 / N$ comes from $\Delta y_{j}$.) More generally, let there be $r \kappa_{j}^{\prime}$ of all order $\mathcal{O}(1)$ or $\mathcal{O}(N)$. Then, the form factor will be of order $N$ to the power of $r(1-1 / \lambda)-(2 / \lambda) r(r-1) / 2=r(1-r / \lambda)$. Therefore, if the $\lambda>r$ the form factor diverges. In other words such configuration is favorable. This is an exotic violation of the super selection rule since $r$ quasiholes are stuck together. However, the net contributions of these terms are again vanishingly small except, of course, in the long-wavelength limit where only these exceptional states survive.

Almost identical method can be applied to obtain Eq. (6.5). When the limit $x, t \rightarrow 0$ is taken, the propagator becomes just the static density $\rho_{0}$. This gives a generalization of the Selberg's integral formula and might have connections with the $q$-deformed Lie algebra.

\section{APPENDIX B: ASYMPTOTIC EXPANSION}

\section{Dynamical density-density correlation function}

A method for expanding the dynamical density-density correlation functions as $x \rightarrow \infty$ is presented in the appendix. A similar method has been used previously in [18 for the equal-time correlation functions.

The form factor to the DDDCF is largest near a phase space region where all $x_{j}$ 's near zero and $y_{i}$ 's near zero or one. Thus, the integrand will be expanded about this region. In 
Fig. 2 the shaded region gives non-zero contributions to the DDDCF and the regions with darker shades give largest contributions to the DDDCF in the long-wavelength limit. Each of these dark regions is labeled with the index $n$ and is called $n$th sector, whose contribution to the correlation function has a characteristic oscillation, and is spanned by all $x_{i}$ 's close to zero and $n(p-n) y_{j}$ 's close to one (zero).

To leading order in the normalized momentum variables the following relation hold true

$$
Q x \pm E t=2 \pi \rho_{0} \xi^{\mp}\left(\sum_{i=1}^{q} x_{i}+\sum_{j=1}^{p-n} y_{j}\right)-2 \pi \rho_{0} \xi^{ \pm} \sum_{k=1}^{n} w_{k}+2 \pi \rho_{0} n x,
$$

where $\xi^{ \pm}=x \mp v_{s} t$. The sound velocity $v_{s}$ is $2 \pi \rho_{0} \lambda$. A new set of $n$ variables $w_{k}$ for $y_{j}$ close to one are introduced such that $w_{k}=1-y_{j} \cdot \xi^{+}\left(\xi^{-}\right)$is the space-time coordinate for the right (left) moving particle or hole excitations.

To leading order in each of the $p$ sectors the DDDCF is given by

$$
\begin{aligned}
\langle\rho(x, t) \rho(0,0)\rangle & \approx \frac{C}{2}\left(I_{1}^{\lambda}(+i \mid q, p)\left(\frac{1}{\xi^{-}}\right)^{2}+I_{1}^{\lambda}(-i \mid q, p)\left(\frac{1}{\xi^{+}}\right)^{2}\right) \\
& +\sum_{n=1}^{p} \tilde{\mathcal{C}}_{n}\left(\frac{1}{\xi^{+} \xi^{-}}\right)^{\frac{n^{2}}{\lambda}} \frac{1}{2}\left(I_{1}^{\lambda}(-i \mid q, p-n) I_{2}^{\lambda}(+i \mid n) e^{i 2 \pi \rho_{0} n x}\right. \\
& \left.+I_{1}^{\lambda}(i \mid q, p-n) I_{2}^{\lambda}(-i \mid n) e^{-i 2 \pi \rho_{0} n x}\right)
\end{aligned}
$$

where $\tilde{\mathcal{C}}_{n}=\left(\begin{array}{l}p \\ n\end{array}\right) C \lambda^{-2 n q} n^{2}\left(\frac{1}{2 \pi \rho_{0}}\right)^{\frac{2 n^{2}}{\lambda}-2}$ and the functions $I_{1}^{\lambda}$ and $I_{2}^{\lambda}$ are defined as follow

$$
\begin{aligned}
I_{1}^{\lambda}(z \mid l, p-m) & =\prod_{i=1}^{l} \int_{0}^{\infty} d x_{i} \prod_{j=1}^{p-m} \int_{0}^{\infty} d y_{j}\left\{\begin{array}{cc}
\left(\sum_{i=1}^{l} x_{i}+\sum_{j=1}^{p} y_{j}\right)^{2} & \text { if } m=0 \\
1 & \text { if } m \neq 0
\end{array}\right\} \\
& \times \prod_{i=1}^{l} \prod_{j=1}^{p-m}\left(x_{i}+\lambda y_{j}\right)^{-2} \frac{\prod_{i<j}\left|x_{i}-x_{j}\right|^{2 \lambda} \prod_{i^{\prime}<j^{\prime}}\left|y_{i^{\prime}}-y_{j^{\prime}}\right|^{2 / \lambda}}{\prod_{i=1}^{l} x_{i}^{1-\lambda} \prod_{j=1}^{p-m} y_{j}^{1-1 / \lambda}} \\
& \times \exp \left(-z\left(\sum_{i} x_{i}+\sum_{j} y_{j}\right)\right) \\
I_{2}^{\lambda}(z \mid m) & =\prod_{k=1}^{m} \int_{0}^{\infty} d w_{k} \frac{\prod_{i<j}\left|w_{i}-w_{j}\right|^{2 / \lambda}}{\prod_{k=1}^{m} w_{k}^{1-1 / \lambda}} \exp \left(-z \sum_{k} w_{k}\right)
\end{aligned}
$$

Since $I_{1}^{\lambda}$ and $I_{2}^{\lambda}$ are absolutely convergent only if $\operatorname{Re}(z)>0$, it is necessary to analytically continue the functions as follow 


$$
\begin{aligned}
I_{1}^{\lambda}(z \mid l, m) & =\left(\frac{1}{z}\right)^{\lambda l^{2}+m^{2} / \lambda-2 l m+2 \delta_{m, p}} I_{1}^{\lambda}(1 \mid l, m), \\
I_{2}^{\lambda}(z \mid m) & =\left(\frac{1}{z}\right)^{m^{2} / \lambda} I_{2}^{\lambda}(1 \mid m) .
\end{aligned}
$$

The above analytical extensions give the following relations: $I_{1}^{\lambda}(-i \mid q, p-n) I_{2}^{\lambda}(+i \mid n)=$ $I_{1}^{\lambda}(1 \mid q, p-n) I_{2}^{\lambda}(1 \mid n)$ and $I_{1}^{\lambda}( \pm i \mid q, p)=-I_{1}^{\lambda}(1 \mid q, p)$.

Finally, the DDDCF to leading order in each harmonic mode is given by

$$
\langle 0|\rho(x, t) \rho(0,0)| 0\rangle \approx-\frac{C}{2} I_{1}^{\lambda}(1 \mid q, p)\left(\frac{1}{\left(\xi^{+}\right)^{2}}+\frac{1}{\left(\xi^{-}\right)^{2}}\right)+\sum_{n=1}^{p} \mathcal{C}_{n}\left(\frac{1}{\xi^{+} \xi^{-}}\right)^{n^{2} / \lambda} \cos \left(2 \pi \rho_{0} n x\right),
$$

where $\mathcal{C}_{n}=\tilde{\mathcal{C}}_{n} I_{1}^{\lambda}(1 \mid q, p-n) I_{2}^{\lambda}(1 \mid n)$.

The renormalization parameter is defined in 499 as $\eta=2\left(v_{J} / v_{N}\right)^{1 / 2}$, where $v_{J}$ and $v_{N}$ are the current and charge velocities. For the $U(1) \operatorname{CSM} \eta=2 / \lambda[10$. 2. With this identification the form of Eq. (B7) in the static limit agrees with the expansion given in 49]. Furthermore, by matching the universal constant in the 0th sector I deduce the following integral formula

$$
I_{1}^{\lambda}(1 \mid q, p)=\left(2 \pi^{2} \lambda C\right)^{-1}
$$

\section{One-particle Green's function}

Using the similar method used for the DDDCF I find the following leading order terms for the hole propagator

$$
\left\langle 0\left|\Psi^{\dagger}(x, t) \Psi(0,0)\right| 0\right\rangle \approx \sum_{n=0}^{p} \mathcal{D}_{n}\left(\frac{1}{2 \pi \rho_{0} \xi^{+}}\right)^{(n-\lambda)^{2} / \lambda}\left(\frac{1}{2 \pi \rho_{0} \xi^{-}}\right)^{n^{2} / \lambda} e^{i\left(2 \pi \rho_{0}(n-\lambda / 2) x+\left(\pi \rho_{0} \lambda\right)^{2} t\right)}
$$

where $\mathcal{D}_{n}=\rho_{0} D \lambda^{1-\lambda-2(q-1) n}\left(\begin{array}{c}p \\ n\end{array}\right) I_{1}^{\lambda}(1 \mid q-1, p-n) I_{2}^{\lambda}(1 \mid n) e^{-i \pi(n-\lambda / 2)}$. Here, $I_{1}^{\lambda}(z \mid q-1, p)$ is defined as before but without the pre-factor $\left(\sum_{i} x_{i}+\sum_{j} y_{j}\right)^{2}$.

\footnotetext{
${ }^{2}$ The interaction coupling constant $\lambda$ used in Ref. [10] corresponds to $\lambda-1$ in this paper.
} 


\section{REFERENCES}

[1] M. G. G. Laidlaw and C. M. DeWitt, Phys. Rev. D 3, 1375 (1971); D. Finkelstein and J. Rubinstein, J. Math. Phys. 9, 1762 (1968).

[2] J. M. Leinaas and J. Myrheim, Nuovo Cimento B 37, 132 (1977).

[3] K. von Klitzing, G. Dorda, and M. Pepper, Phys. Rev. Lett. 45494 (1980); D. C. Tsui, H. L. Stormer, and A. C. Gossard, Phys. Rev. Lett. 48, 1559 (1982).

[4] F. Calogero, J. Math. Phys. 10,2191 (1969); B. Sutherland, Phys. Rev. A4, 2019 (1971).

[5] F. D. M. Haldane, in the Proceedings of the 16th Taniguchi Symposium, Kashikijima, Japan, October 26-29, 1993, eds. A. Okiji and N. Kawakami, Springer-Verlag, 1994.

[6] Z. N. C. Ha, Phys. Rev. Lett. 731574 (1994).

[7] F. D. M. Haldane, Phys. Rev. Lett. 60, 635 (1988); B. S. Shastry, ibid. 60, 639 (1988).

[8] F. D. M. Haldane, Z. N. C. Ha, J. C. Talstra, D. Bernard, and V. Pasquier, Phys. Rev. Lett. 69, 2021 (1992).

[9] F. D. M. Handane, Phys. Rev. Lett. 66, 1529 (1991).

[10] Z. N. C. Ha an F. D. M. Haldane, Bull. Am. Phys. Soc. 37, 646 (1992); Phys. Rev. B 46, 9359 (1992).

[11] N. Kawakami, Phys. Rev. B 46, 1005 (1992); 46, 3192 (1992).

[12] Z. N. C. Ha and F. D. M. Haldane, Phys. Rev. B 47, 12459 (1993).

[13] F. J. Dyson, J. Math. Phys. 3, 140 (1962); 157 (1962); 166 (1962).

[14] M. L. Mehta, Random Matrices, 2nd Ed., Academic Press, Inc., 1991; F. J. Dyson, J. Math. Phys. 19235 (1970).

[15] K. B. Efetov, Adv. Phys. 32, 53 (1983). 
[16] B. D. Simons, P. A. Lee, and B. L. Altshuler, Phys. Rev. Lett. 70, 4122 (1993).

[17] F. D. M. Haldane and M. R. Zirnbauer, Phys. Rev. Lett. 71, 4055 (1993).

[18] P. J. Forrester, Phys. Lett. A 179, 127 (1993); Nucl. Phys. B 388, 671 (1992).

[19] J. Kaneko, SIAM J. Math. Anal. 24, 1086 (1993); K. W. J. Kadell, Adv. Math. (to be published).

[20] P. J. Forrester, Nucl. Phys. B416, 377 (1994).

[21] F. D. M. Haldane, Bull. Am. Phys. Soc. 37, 164 (1992); Phys. Rev. Lett. 67, 937 (1991);

S. Mitra and A. H. MacDonald, ibid. 37, 377 (1992); P. J. Forrester and B. Jancovici, J. Phys. (Paris) 45, L583 (1984); A. P. Polychronakos, Nucl. Phys. B324, 597 (1989); N. Kawakami, Phys. Rev. Lett. 71, 275 (1993); S. Iso and S. J. Rey, (unpublished); A. D. de Veigy and S. Ouvry, Phys. Rev. Lett. 72, 121 (1994).

[22] B. Sutherland, Phys. Rev. A5, 1372 (1972).

[23] R. B. Laughlin, Phys. Rev. Lett. 50, 1395 (1983).

[24] X. G. Wen, Phys. Rev. Lett. 64, 216 (1990), Phys. Rev. B 41, 12838 (1990); Mod. Phys. Lett. B 5, 39 (1991).

[25] H. Jack, Proc. Roy. Soc. Edinburgh Sect. A 691 (1969-1970).

[26] R. P. Stanley, Adv. Math. 77, 76 (1989).

[27] I. G. Macdonald, Symmetric Functions and Hall Polynomials, Oxford Univ. Press, Oxford, 1979.

[28] Ref. [27], 2nd ed. (to appear).

[29] I. G. Macdonald, Lecture Notes in Math. 1271, 189 (1987).

[30] K. W. J. Kadell, Compos. Math. 87, 5 (1993). 
[31] P. J. Hanlon, R. P. Stanley, and J. R. Stembridge, Contemp. Math. 138, 151 (1992).

[32] Z. Yan, Contemp. Math. 138, 239 (1992).

[33] See, for example, the articles in R. E. Prange and S. M. Girvin eds., The Quantum Hall Effect, Springer-Verlag, 1987; M. Stone ed., The Quantum Hall Effect, World Scientific, Singapore, 1992.

[34] J. Frohlich, in Nonperturbative Quantum Field theory, NATO ASI Series B: Physics Vol. 185, $71(1987)$.

[35] M. Jimbo ed., Yang-Baxter Equation in Integrable Systems, Advanced Series in Math. Phys. Vol. 10, World Scientific, Singapore, 1989; T. Kohno ed., New Developments in the Theory of Knots, ibid. Vol. 11; L. H. Kauffman, Knots and Physics, World Scientific, Singapore, 1991.

[36] A. P. Polychronakos, Phys. Rev. Lett. 69, 703 (1992).

[37] C. N. Yang and C. P. Yang, J. Math. Phys. 10, 1115 (1969).

[38] D. Bernard and Y. S. Wu, (unpublished).

[39] Y. S. Wu, Phys. Rev. Lett. 73, 922 (1994).

[40] F. D. M. Haldane, in the proceeding of the International Colloquium in Modern Field Theory, Tata Institute, Bombay, India, January 5-12, 1994.

[41] F. Lesage, V. Pasquier, and D. Serban (unpublished); P. J. Forrester (unpublished); J. Minahan and A. P. Polychronakos, Phys. Rev. B50, 4236 (1994).

[42] D. J. Gross and A. Matytsin, (unpublished); and references therein.

[43] A. Lenard, J. Math. Phys. 5, 930 (1964).

[44] F. D. M. Haldane, J. Phys. C14 2585 (1981); A. Luther and I. Peschel, Phys. Rev. B9, 2911 (1974); E. H. Lieb and D. C. Mattis, J. Math. Phys. 6304 (1965); J. M. Luttinger, 
J. Math. Phys. 41154 (1963);

[45] See, for example, Quantum Field Theory and Critical Phenomena, Clarendon Press (Oxford), 1989.

[46] R. Tao and Y. S. Wu, Phys. Rev. B30, 1097 (1984); R. B. Laughlin, Phys. Rev. B23, 5632 (1981); B. I. Halperin, Phys. Rev. B25, 2185 (1982).

[47] E. Fradkin, Field Theories of Condensed Matter Physics, Addison-Wesley, 1991; First Ref. in [21].

[48] L. P. Kadanoff and H. Ceva, Phys. Rev. B3, 3918 (1971); E. Fradkin and L. Susskind, Phys. Rev. D17, 2637 (1978).

[49] F. D. M. Haldane, Phys. Rev. Lett. 47, 1840 (1982).

[50] G. D. Mahan, Many-Particle Physics, Plenum, New York, 2nd ed., 1990.

[51] J. L. Cardy, Nucl. Phys. B270[FS16], 186 (1986).

[52] N. Kawakami and S. K. Yang, Phys. Rev. Lett. 67, 2493 (1991). 


\section{FIGURES}

FIG. 1. A level diagram for a family with the highest-level mother-state given by $|6\rangle_{1}=\Phi(4,3,1,0)$. The arrows connect the mother states to their daughter states that are generated by squeezing on the pairs of quantum numbers by one unit.

FIG. 2. The shaded area is the region of support for the dynamical density-density correlation function at $\lambda=p / q=5 / 3$ in the energy-momentum space. The energy $E$ is in some arbitrary unit and the momentum $Q$ in units of $2 \pi \rho_{0}$. The $2 p$ distinct regions with darker shade corresponds to the low-energy sectors of the model.

FIG. 3. Cylinderical geometry for the anyon system. Two oppositely-moving edges along $x$-axis are separated by an incompressible anyon fluid. The cylinder is pierced by a solenoid which induces a magnectic flux $\Phi$ through its cross-section.

FIG. 4. A diagram $\mathcal{D}$ that contributes to the DDDCF at $\lambda=p / q=5 / 6$ is divided into three subdiagrams $\mathcal{A}, \mathcal{B}$, and $\mathcal{C}$ for convienience.

FIG. 5. A subdiagram $\mathcal{B}$ with $p=10$ is divided into $p$ cells. The dots $\bullet$ indicate the empty cells where $\kappa_{l}^{\prime}=\kappa_{l+1}^{\prime}$, and only the $1,3,4,5,8$, and 10 th cells are non-empty. For the $l$ th cell, $\kappa_{i}$ are all same and equal to $l$. 
Fig. 1

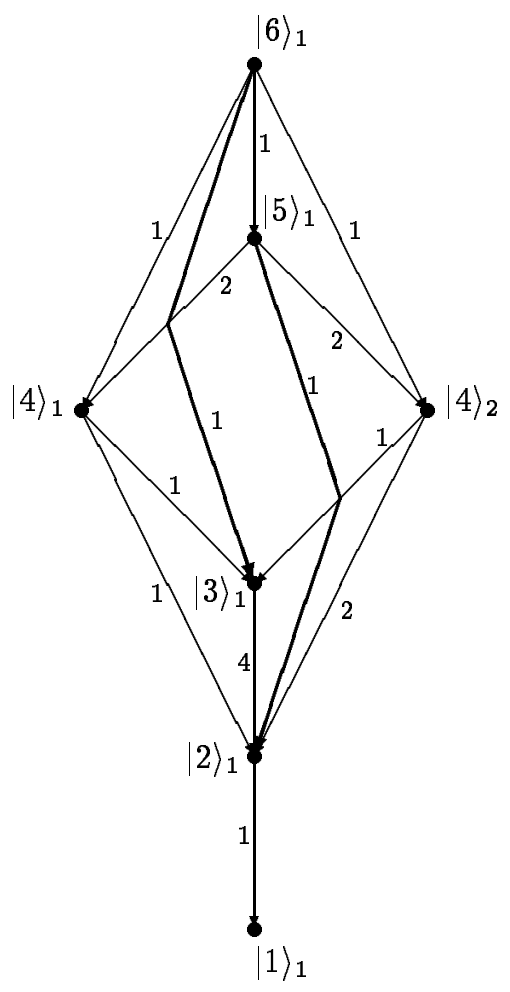




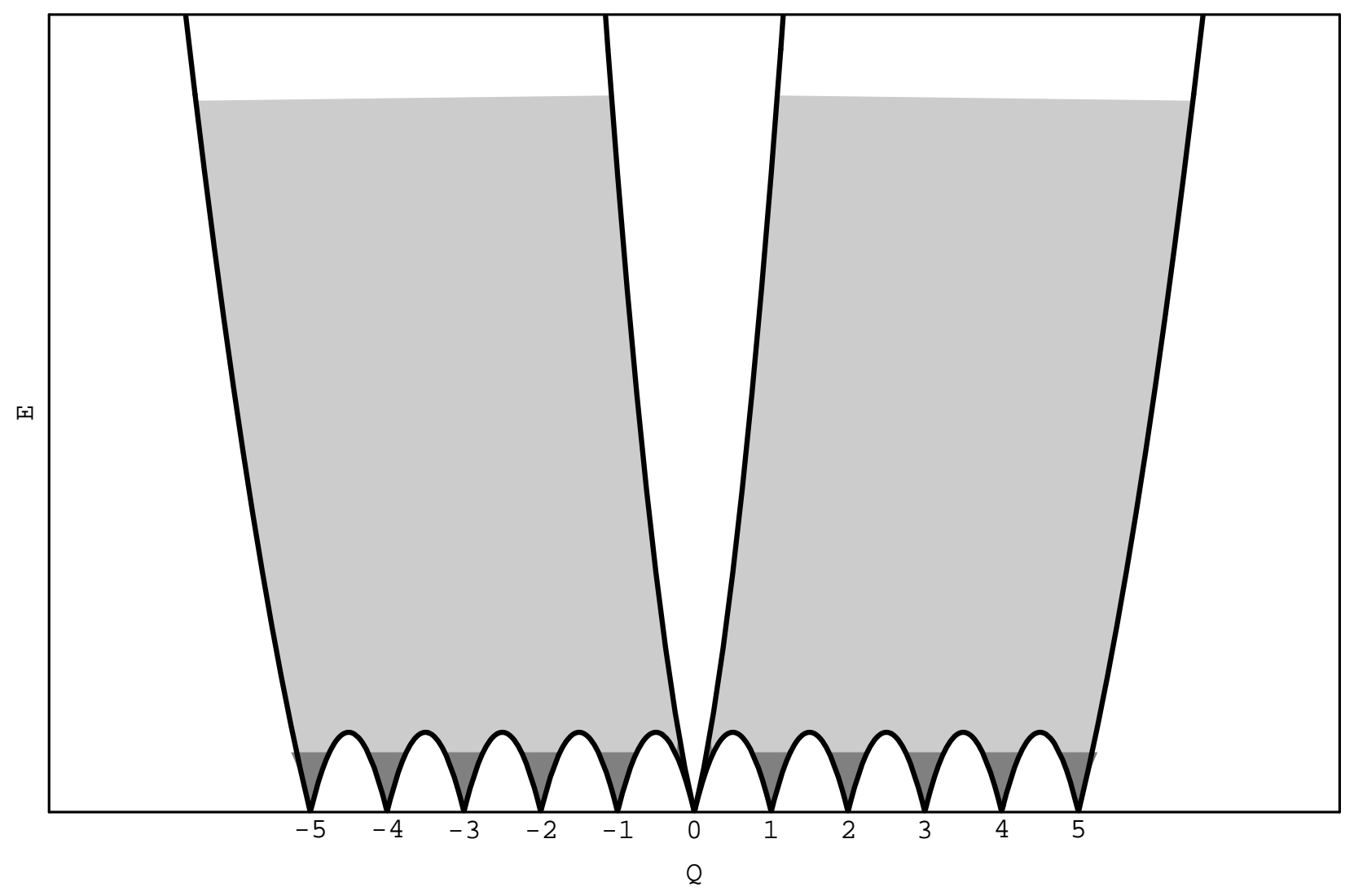




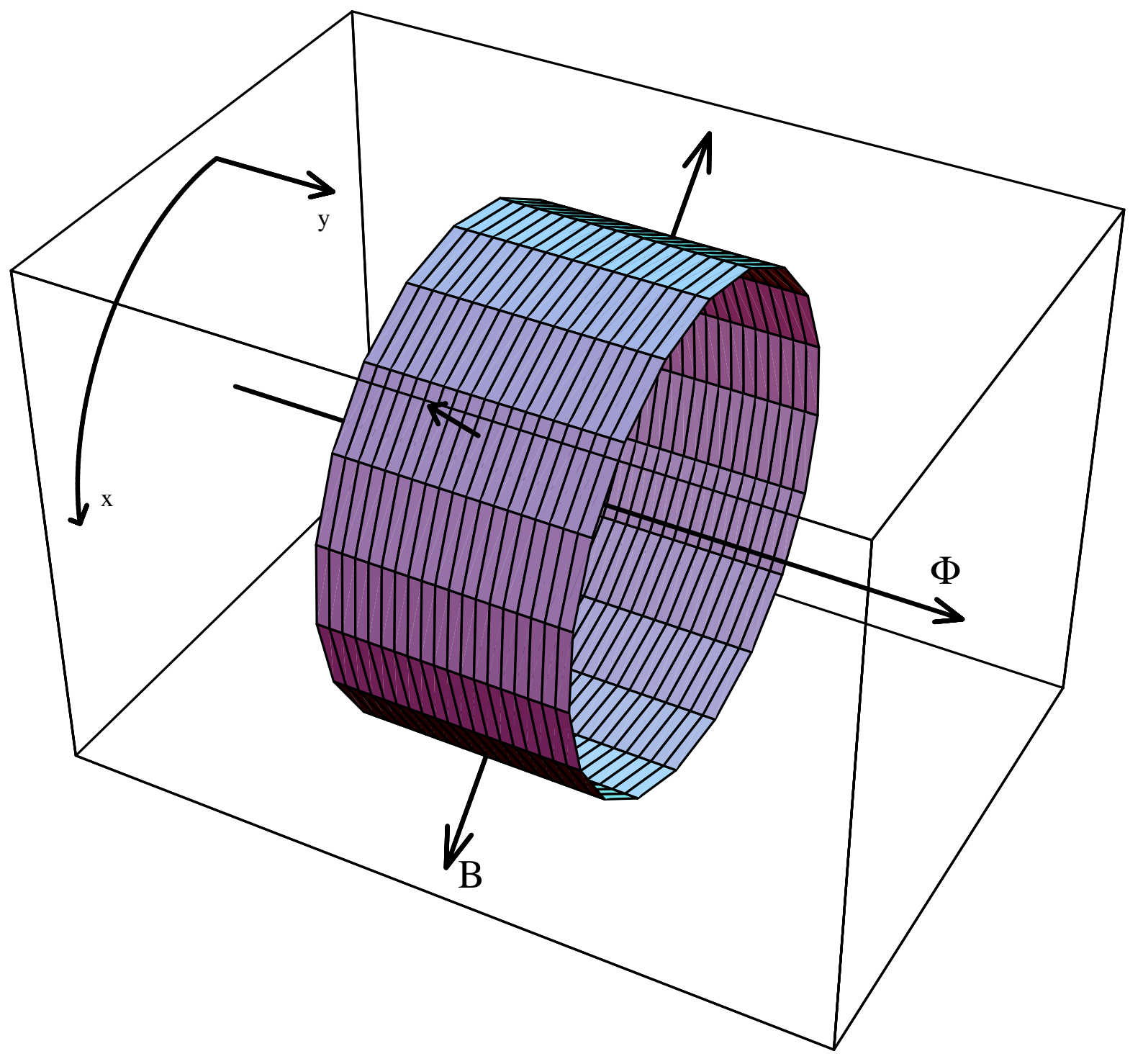


Fig. 4

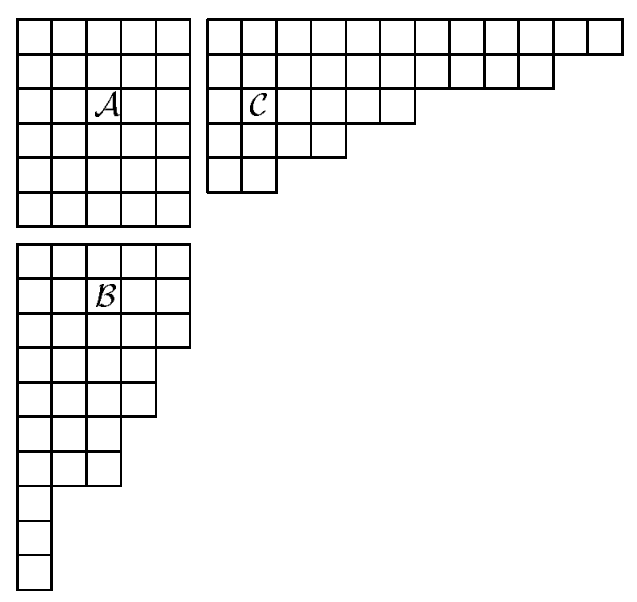


Fig. 5

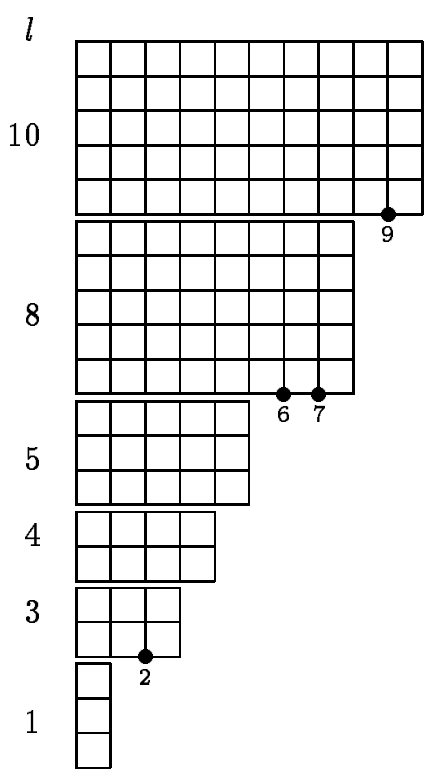

